\title{
A role for the cortex in sleep-wake regulation
}

\section{Journal Article}

\section{Author(s):}

Krone, Lukas B.; Yamagata, Tomoko; Blanco-Duque, Cristina; Guillaumin, Mathilde C.C.; Kahn, Martin C.; van der Vinne, Vincent; McKillop, Laura E.; Tam, Shu K. E.; Peirson, Stuart N.; Akerman, Colin J.; Hoerder-Suabedissen, Anna; Molnar, Zoltan; Vyazovskiy, Vladyslav V.

\section{Publication date:}

2021-09

\section{Permanent link:}

https://doi.org/10.3929/ethz-b-000501216

\section{Rights / license:}

Creative Commons Attribution 4.0 International

\section{Originally published in:}

Nature Neuroscience 24, https://doi.org/10.1038/s41593-021-00894-6 


\title{
A role for the cortex in sleep-wake regulation
}

\author{
Lukas B. Krone ${ }^{1,2}$, Tomoko Yamagata ${ }^{2,3}$, Cristina Blanco-Duque ${ }^{1,2,4}$, Mathilde C. C. Guillaumin ${ }^{2,3,5}$, \\ Martin C. Kahn 1,2,4, Vincent van der Vinne, ${ }^{1,2,6}$, Laura E. McKillop ${ }^{1,2}$, Shu K. E. Tam ${ }^{2,3}$, \\ Stuart N. Peirson ${ }^{2,3}$, Colin J. Akerman $\mathbb{1}^{7}$, Anna Hoerder-Suabedissen ${ }^{\circledR 1}$, Zoltán Molnár ${ }^{1}{ }^{\circledR}$ and \\ Vladyslav V. Vyazovskiy ${ }^{1,2} \bowtie$
}

\begin{abstract}
Cortical and subcortical circuitry are thought to play distinct roles in the generation of sleep oscillations and global state control, respectively. Here we silenced a subset of neocortical layer 5 pyramidal and archicortical dentate gyrus granule cells in male mice by ablating SNAP25. This markedly increased wakefulness and reduced rebound of electroencephalographic slow-wave activity after sleep deprivation, suggesting a role for the cortex in both vigilance state control and sleep homeostasis.
\end{abstract}

The duration, timing and architecture of sleep are strictly regulated. Early studies based on neurological case reports, transections and electrical stimulation suggested that global state transitions are mediated via a distributed circuitry across the brain stem, the hypothalamus and the basal forebrain. More recent studies using selective targeting of specific neuronal populations based on their gene expression or connectivity patterns, highlighted that the sleep-wake promoting circuitry is highly complex, with distinct subcortical brain regions and neuronal subtypes responsible for specific aspects of wakefulness and sleep ${ }^{1,2}$. Although sleep-wake states are defined by the occurrence of neocortical and hippocampal oscillations, the possibility that neocortical and archicortical neurons control vigilance states has been overlooked.

Cortical oscillations and neuronal firing patterns mirror sleep homeostasis ${ }^{3,4}$. Sleep homeostasis refers to the adjustment of the duration and intensity of sleep, to the duration of preceding wakefulness ${ }^{4}$. Electroencephalogram (EEG) slow-wave activity (SWA; EEG spectral power of $0.5-4 \mathrm{~Hz}$ ) during non-rapid eye movement (NREM) sleep represents a reliable marker of sleep-wake history ${ }^{4}$ and has been proposed to underlie many functions of sleep, such as cellular maintenance and synaptic plasticity. SWA can be regulated in a local, use-dependent manner ${ }^{5,6}$, in line with the view that sleep emerges within cortical networks driven by the local accumulation of metabolic products, such as adenosine ${ }^{7}$. However, slow waves also occur under anesthesia, in isolated cortical slabs or even ex vivo $^{8}$. Therefore, the capacity to produce slow waves does not automatically imply a causative role for the cortex in physiological sleep or sleep homeostasis, on either a local or a global level.

Here we test whether cortical structures have a function in regulating global sleep-wake dynamics. We focused on pyramidal neurons within layer 5 of the neocortex as well as archicortical dentate gyrus granule cells, two cell types involved in the generation of sleep oscillations. Layer 5 pyramidal neurons have been shown to be essential for the initiation and propagation of neocortical slow waves $^{9-11}$, the main type of NREM sleep oscillations. Dentate gyrus granule cells contribute to the generation of hippocampal theta rhythm ${ }^{12}$, the defining oscillatory activity of rapid eye movement (REM) sleep in rodents ${ }^{13}$. Yet neither of these two neocortical and archicortical cell populations has so far been implicated in sleepwake control.

Laminar local field potential (LFP) and multiunit activity (MUA) recordings were performed from the primary motor cortex of male adult wild-type (C57BL/6) mice, concomitantly with EEG and electromyography (EMG) monitoring during $24 \mathrm{~h}$ of undisturbed conditions (Fig. 1a-e). Consistent with the idea of an active role for layer 5 in generating slow waves ${ }^{9,10,14}$, we found that neurons in layer 5 tended to initiate spiking upon the onset of population ON periods (Fig. 1e and Extended Data Fig. 1). A leading role for layer 5 was indicated by a stronger initial surge of neuronal firing at OFF-ON transitions (Extended Data Fig. 1a), and a shorter latency to the first spike during ON periods, even when the total number of spikes in layer 5 was matched with that of layer 2/3 (Extended Data Fig. 1b).

To induce a cortex-wide reduction in the output from layer 5 pyramidal neurons, we used a transgenic mouse line, in which a subpopulation $(\sim 15-30 \%)$ of pyramidal cells in layer 5 of the neocortex lack the key t-SNARE (target membrane soluble $\mathrm{N}$-ethylmaleimide-sensitive factor attachment protein receptors) protein SNAP25 (synaptosomal-associated protein $25 \mathrm{kDa}$; Rbp4-Cre;Ai14;Snap2 $\left.5^{\mathrm{f} / \mathrm{f} /}\right)^{15}$. Rbp4-Cre is known as a pan layer 5 driver line (Fig. 1f and Supplementary Fig. 1) ${ }^{16}$, but also presents a strong Cre expression in dentate gyrus granule cells (Extended Data Fig. 2). By contrast, Cre expression outside of cortex is very sparse. Hypothalamic nuclei with established roles in sleep and circadian regulation show no or very few Cre-expressing cells and we found no overlap with orexin-concentrating or melanin-concentrating hormone-expressing cells in lateral hypothalamus (Extended Data Figs. 3-5). As has been shown previously, ablation of SNAP25 virtually abolishes calcium-evoked neurotransmitter release from neurons $^{15}$, rendering the cells functionally silent. Importantly, normal brain development, including cortical layering and axonal path finding, has been shown in SNAP25-ablated mice ${ }^{15}$. We chose this conditional knockout ( $\mathrm{cKO}$ ) mouse to probe a role for the cortex in sleep-wake regulation because in this model large and widely distributed populations of neocortical and archicortical neurons involved in the generation of sleep oscillations and in the communication between cortex and subcortical structures are functionally silenced.

'Department of Physiology, Anatomy and Genetics, University of Oxford, Oxford, UK. ${ }^{2}$ Sleep and Circadian Neuroscience Institute, University of Oxford, Oxford, UK. ${ }^{3}$ Nuffield Department of Clinical Neurosciences, University of Oxford, Oxford, UK. ${ }^{4}$ The Picower Institute for Learning and Memory, Massachusetts Institute of Technology, Cambridge, MA, USA. ${ }^{5}$ Institute for Neuroscience, Department of Health Sciences and Technology, ETH, Zurich, Switzerland. ${ }^{6}$ Department of Biology, Williams College, Williamstown, MA, USA. ${ }^{7}$ Department of Pharmacology, University of Oxford, Oxford, UK. 凶e-mail: zoltan.molnar@dpag.ox.ac.uk; vladyslav.vyazovskiy@dpag.ox.ac.uk 


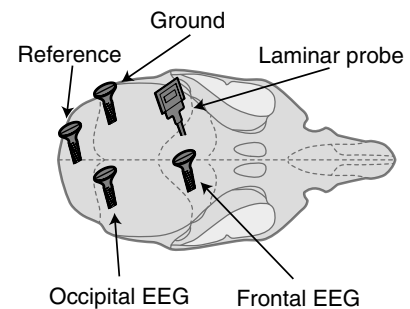

b

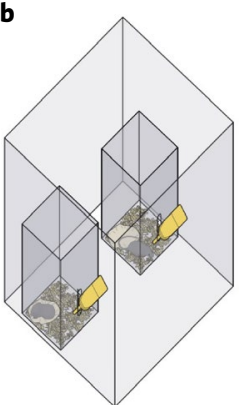

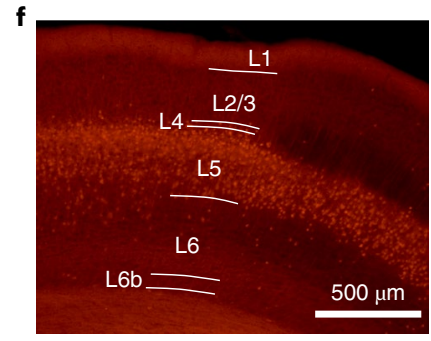

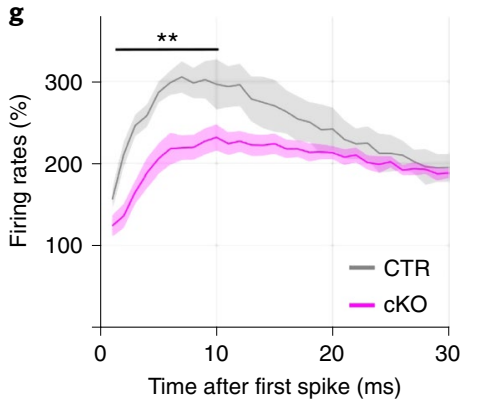

c

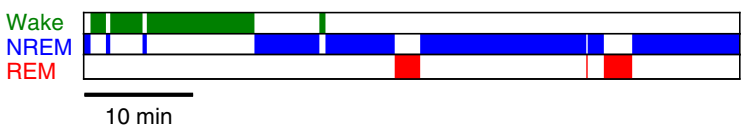

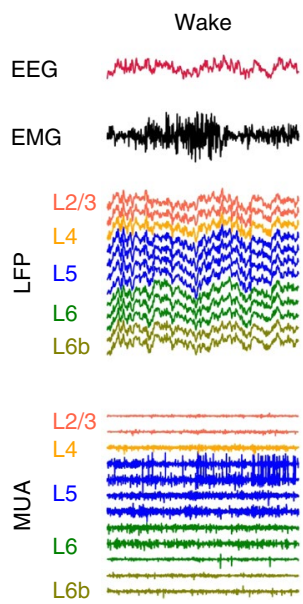

d

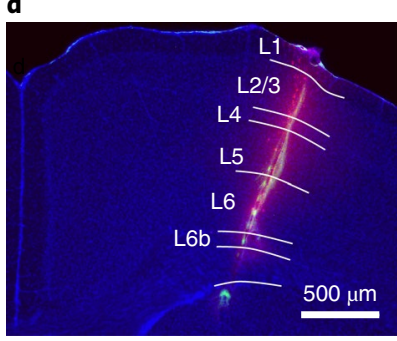

REM
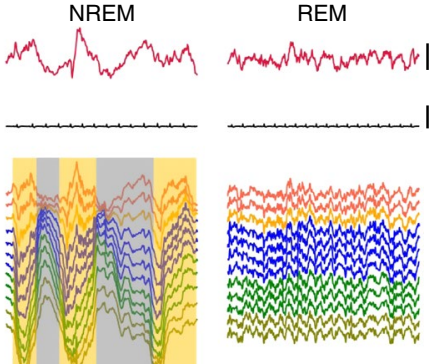

ON OFF
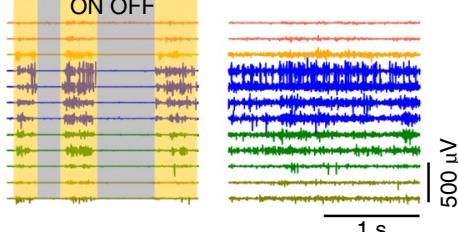

e

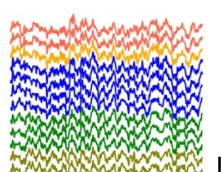

m

h
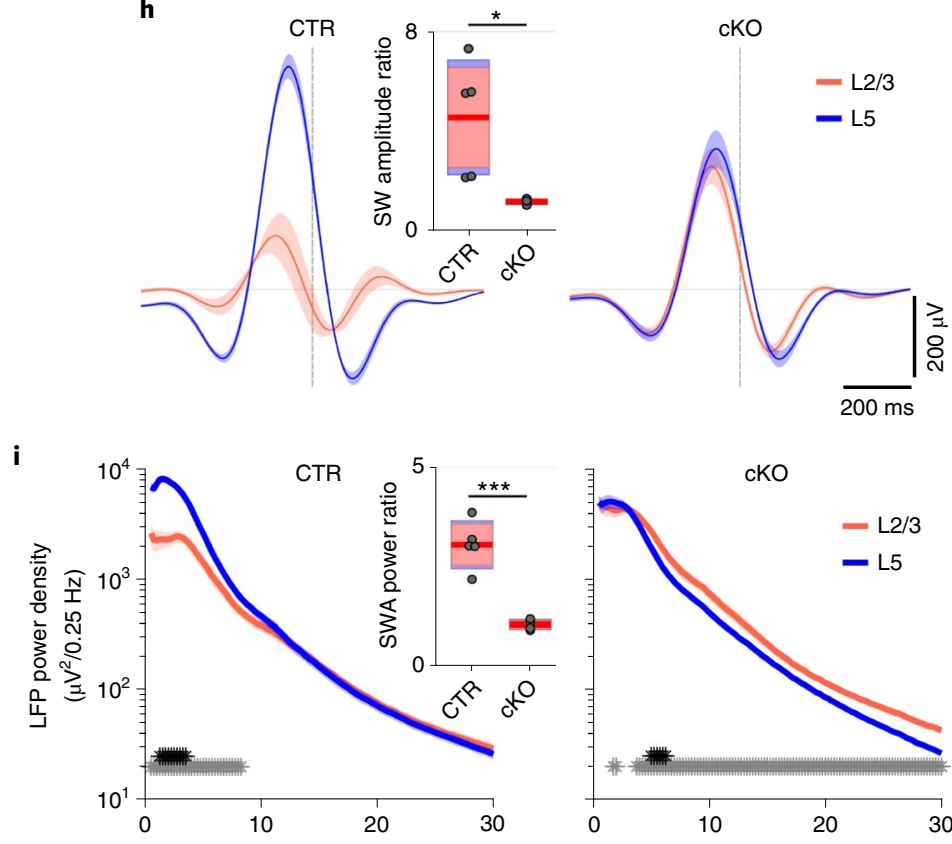

cKO

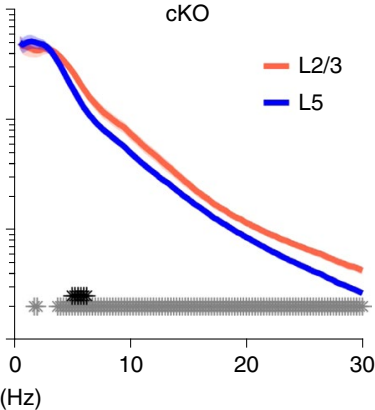

j

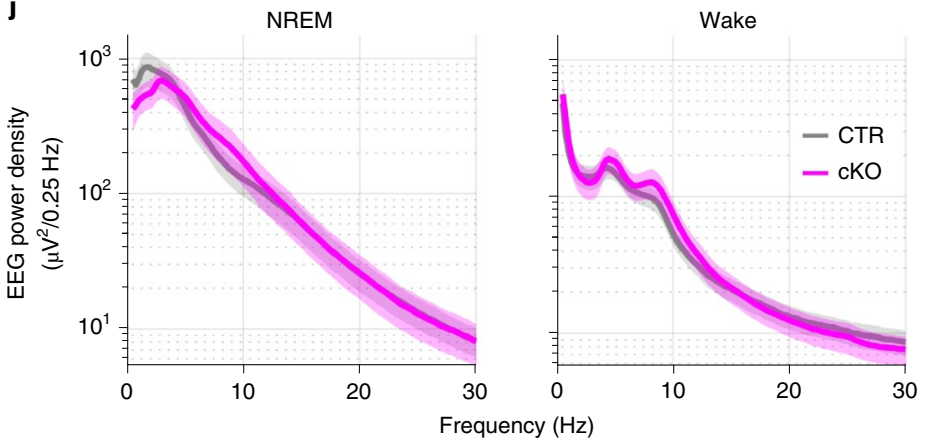

Fig. 1 | Cortical recordings in freely moving mice implicate layer 5 in the generation of slow waves during NREM sleep. a, EEG/LFP electrode positions. b, Schematic of recording setup. c, Representative hypnogram with corresponding EEG, EMG, LFP and MUA traces below. Neuronal ON and OFF periods during NREM sleep are highlighted. Scale bars on the $y$ axis show $500 \mu \mathrm{V}$. d, Insertion tract of a laminar probe. DAPI (4',6-diamidino-2-phenylindole) counterstained (blue) coronal brain section with Dil (1,1'-dioctadecyl-3,3,3',3'-tetramethylindocarbocyanine perchlorate) trace (red). e, Representative OFF-ON transition. Channel assignment and color coding correspond to $\mathbf{c}$. Scale bars on the $y$ axis show $500 \mu \mathrm{V}$. $\mathbf{f}$, Neocortical Cre expression under the Rbp4-Cre promoter. g, Neuronal activity in layer 5 (L5) at OFF-ON transitions in conditional knockout (cKO) and control (CTR) animals relative to NREM sleep average (genotype $\times$ time interaction: $F(29,232)=4.326, P<0.001$, mixed analysis of variance (ANOVA)). $\mathbf{h}$, Average LFP slow wave in layers $2 / 3(\mathrm{~L} 2 / 3)$ and L5 aligned to the OFF-ON transition (vertical dashed line) in CKO and CTR mice (genotype $\times$ layer interaction for slow wave amplitude: $F(1,8)=95.172, P<0.001$, mixed ANOVA). Inset shows the SW amplitude ratio between L2/3 and L5. $\mathbf{i}$, LFP spectra of L2/3 and L5 of cKO and CTR mice during NREM sleep (genotype $\times$ layer interaction for slow wave activity: $F(1,8)=114.820, P<0.001$, mixed ANOVA). Asterisks indicate frequency bins with significant differences in post hoc comparison before (gray) and after (black) Bonferroni adjustment of $\alpha$. Inset shows the SWA ratio between L2/3 and L5. j, Frontal EEG spectra during NREM sleep and wakefulness (genotype $\times$ frequency interaction for NREM sleep: $F(118,1298)=2.793, P<0.001$; genotype $\times$ frequency interaction for wakefulness: $F(118,1298)=1.998, P<0.001$, mixed ANOVAs). Post-hoc comparisons did not reveal significant genotype differences for any of the frequency bins in either of the two vigilance states. $n=5$ CTR and $n=5$ CKO for laminar analysis ( $\mathbf{g}-\mathbf{i}), n=5$ CTR and $n=8 \mathrm{cKO}$ for EEG spectral analysis (j). Black asterisks indicate post hoc contrasts with significant differences $\left({ }^{\star} P<0.05,{ }^{\star \star} P<0.01,{ }^{\star \star \star} P<0.001\right)$. Data in $\mathbf{g}-\mathbf{j}$ are presented as the mean \pm s.e.m. (shaded areas). Insets in $\mathbf{h}$ and $\mathbf{i}$ represent grouped data including the group mean (red line), $95 \%$ confidence interval (pink box) and one standard deviation (blue box) with individual data points overlaid. See Supplementary Table 1 for detailed results. 


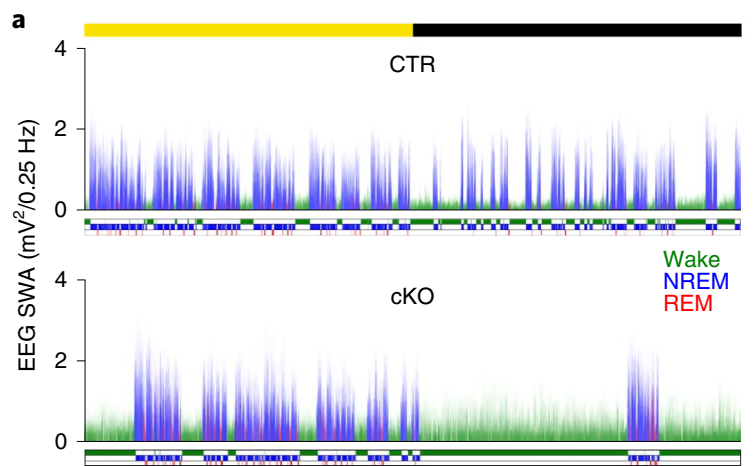

b

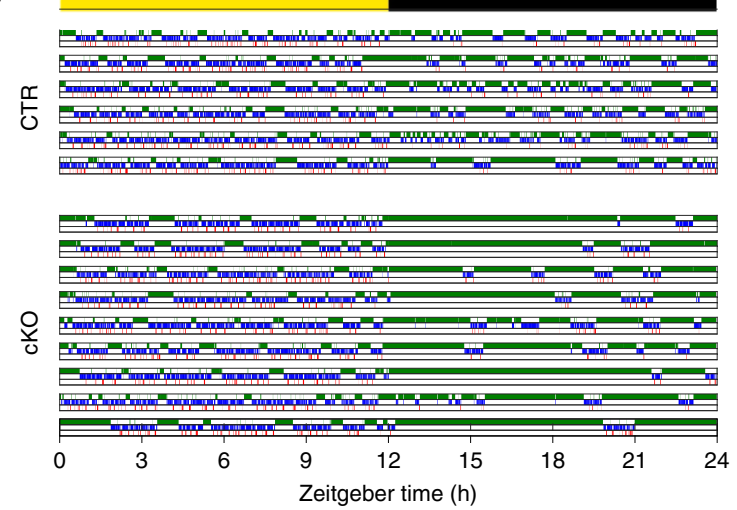

c
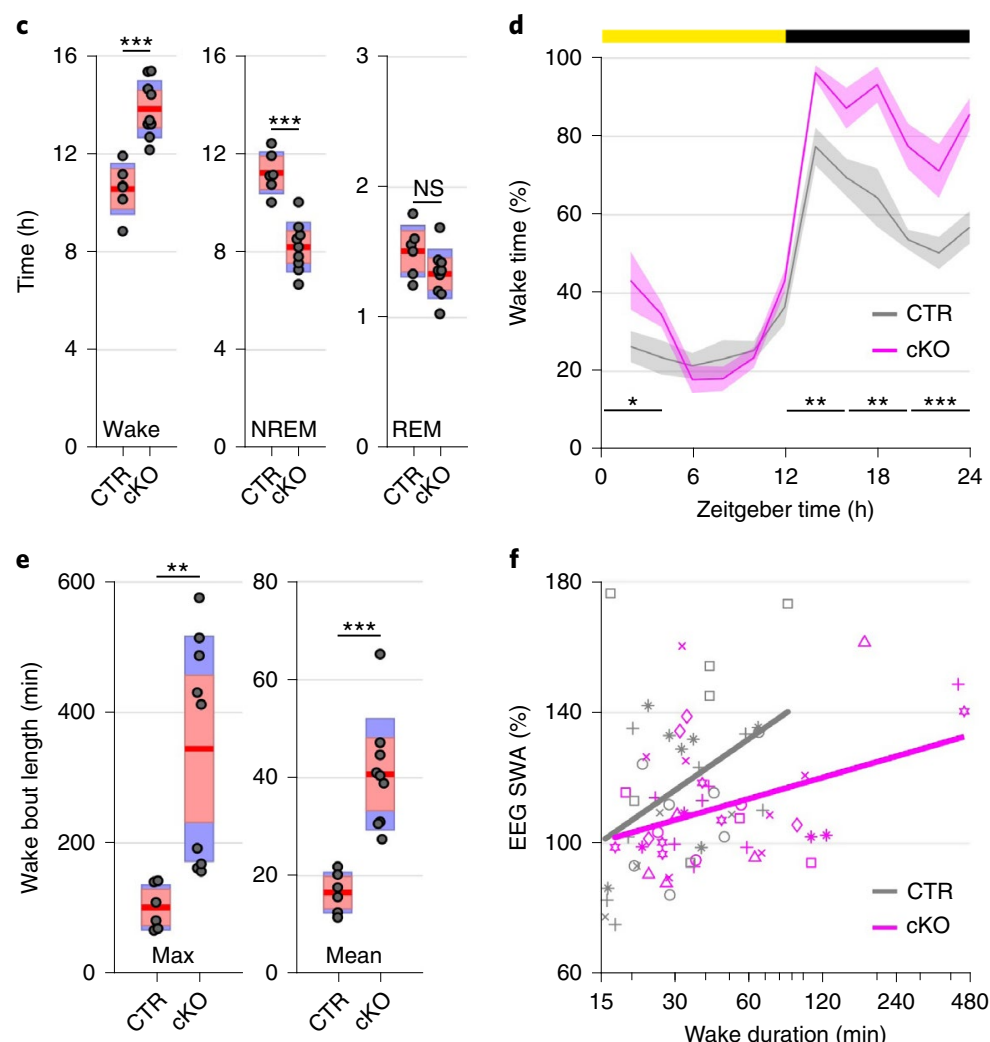

f

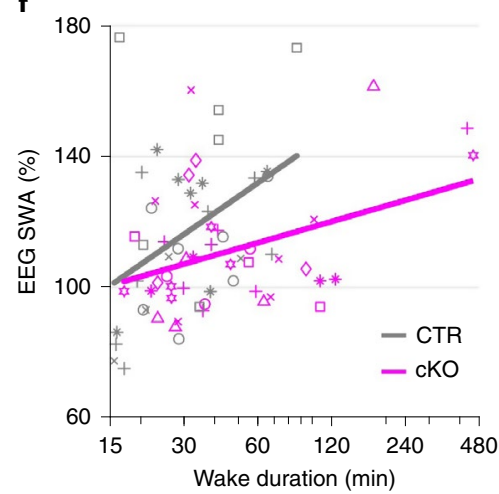

Fig. 2 Selective cortical SNAP25 ablation alters sleep architecture. a, Hypnogram and EEG SWA (0.5-4.0 Hz, 4-s epochs) of one representative animal from each genotype in undisturbed 24-h baseline recordings. $\mathbf{b}$, Individual hypnograms for CTR and cKO animals under undisturbed baseline conditions. Note the increased amount of wakefulness and long wake episodes in CKO mice (wake, green; NREM, blue; REM, red). c, Time spent in vigilance states (wake, NREM and REM) during 24-h baseline recordings (genotype $\times$ vigilance state interaction: $F(1.056,13.732)=33.008, P<0.001$, mixed ANOVA). d, Time course of wakefulness over 24-h baseline recordings (main effect of genotype: $F(1,13)=30.804, P<0.001$, mixed ANOVA). e, Maximum and mean duration of all spontaneous wake episodes over the 2-d recording period, excluding the 6-h sleep deprivation (main effects of genotype: $F(1,13)=11.326$, $P=0.005$ for maximum duration; $F(1,13)=24.392, P<0.001$ for mean duration, two one-way ANOVAs). f, Relationship between wake duration and relative NREM SWA in the frontal EEG derivation during the baseline day. Individual animals are represented with different symbols. $n=6$ CTR and $n=9 \mathrm{cKO}$ for vigilance state analysis ( $\mathbf{c}, \mathbf{d}$ and $\mathbf{e}$ ), $n=5 \mathrm{CTR}$ and $n=8 \mathrm{cKO}$ for EEG spectral analysis (f). Black asterisks indicate post hoc contrasts with significant differences $\left({ }^{\star} P<0.05,{ }^{\star \star} P<0.01,{ }^{\star \star \star} P<0.001\right.$ ), and gray asterisk indicates post hoc comparison with $P<0.05$, which did not reach significance after Bonferroni correction for multiple comparisons. Data in $\mathbf{d}$ are presented as the mean \pm s.e.m. (shaded areas). Yellow and black bars above panels $\mathbf{a}, \mathbf{b}$ and $\mathbf{d}$ indicate light and dark periods, respectively. Data in $\mathbf{c}$ and $\mathbf{e}$ represent grouped data including the group mean (red line), $95 \%$ confidence interval (pink box) and one standard deviation (blue box) with individual data points overlaid. See Supplementary Table 1 for detailed results. NS, not significant.

To investigate sleep architecture and electrophysiology in cortical SNAP25-ablated mice, we performed chronic EEG, LFP and MUA recordings as in wild-type mice. Laminar MUA revealed a diminished surge of layer 5 firing at the onset of population ON periods (Fig. $1 \mathrm{~g}$ ). In addition, there were significant interaction effects between genotype and cortical layer for both slow-wave amplitudes $(F(1,8)=95.17, P<0.001)$ and SWA $(F(1,8)=114.82, P<0.001)$. The amplitude of LFP slow waves in layer 5 was decreased $(t(8)=3.70$, $P=0.006, d=2.34)$, and the levels of SWA were reduced $(t(8)=2.87$, $P=0.021, d=1.81$; Fig. 1h,i). The opposite pattern was observed in layer $2 / 3$, where a trend toward increased slow-wave amplitudes $(t(8)=-2.21, P=0.058, d=-1.40)$ and elevated SWA $(t(8)=3.07$, $P=0.15, d=-1.94$ ) was observed (Fig. 1h,i). The differential regulation of layer 5 and layer $2 / 3$ slow waves in cortical SNAP25-ablated mice resulted in a significantly reduced ratio of slow-wave amplitudes and SWA in cortical SNAP25-ablated mice (Fig. 1h,i). These layer-specific changes to intracortical dynamics are consistent with a reduction of local monosynaptic excitation between layer 5 pyramidal neurons ${ }^{17}$ and diminished disynaptic inhibition of layer $2 / 3$ pyramidal neurons $^{18}$. Laminar firing rates and the latency to the first spike in layer 5 were not significantly altered in cortical
SNAP25-ablated mice, in line with previous reports that abolition of evoked synaptic neurotransmitter release through SNAP25 ablation does not prevent neurons from depolarizing (Supplementary Fig. 2) ${ }^{19}$. The profound layer-specific changes in LFP SWA during NREM sleep contrasted with a lack of major differences in EEG power spectra during NREM sleep or wakefulness (Fig. 1j and Supplementary Fig. 3). However, cortical SNAP25-ablated mice presented a leftward shift of the EEG theta peak frequency during REM sleep (Extended Data Fig. 5). These layer-specific and sleep-state-specific findings underscore the importance of assessing sleep electrophysiology on the local and global levels ${ }^{20}$.

Beyond the local and global changes in sleep oscillations, we observed profound genotype differences in the daily sleep-wake profile (Fig. 2a). While control animals showed sleep architecture typical for wild-type mice (Fig. 2b) ${ }^{21}$, cortical SNAP25-ablated animals presented unusually long wake bouts, often lasting several hours (Fig. 2b,e). On average, cKO mice spent $13.83 \pm 0.39 \mathrm{~h}$ awake per day, approximately $3 \mathrm{~h}$ more than controls $(10.57 \pm 0.42 \mathrm{~h}$; $t(13)=5.55, P<0.001, d=2.96)$, and the amount of sleep decreased proportionally (Fig. 2c). The differences between genotypes were more pronounced in the dark period (Fig. 2d and Extended Data 

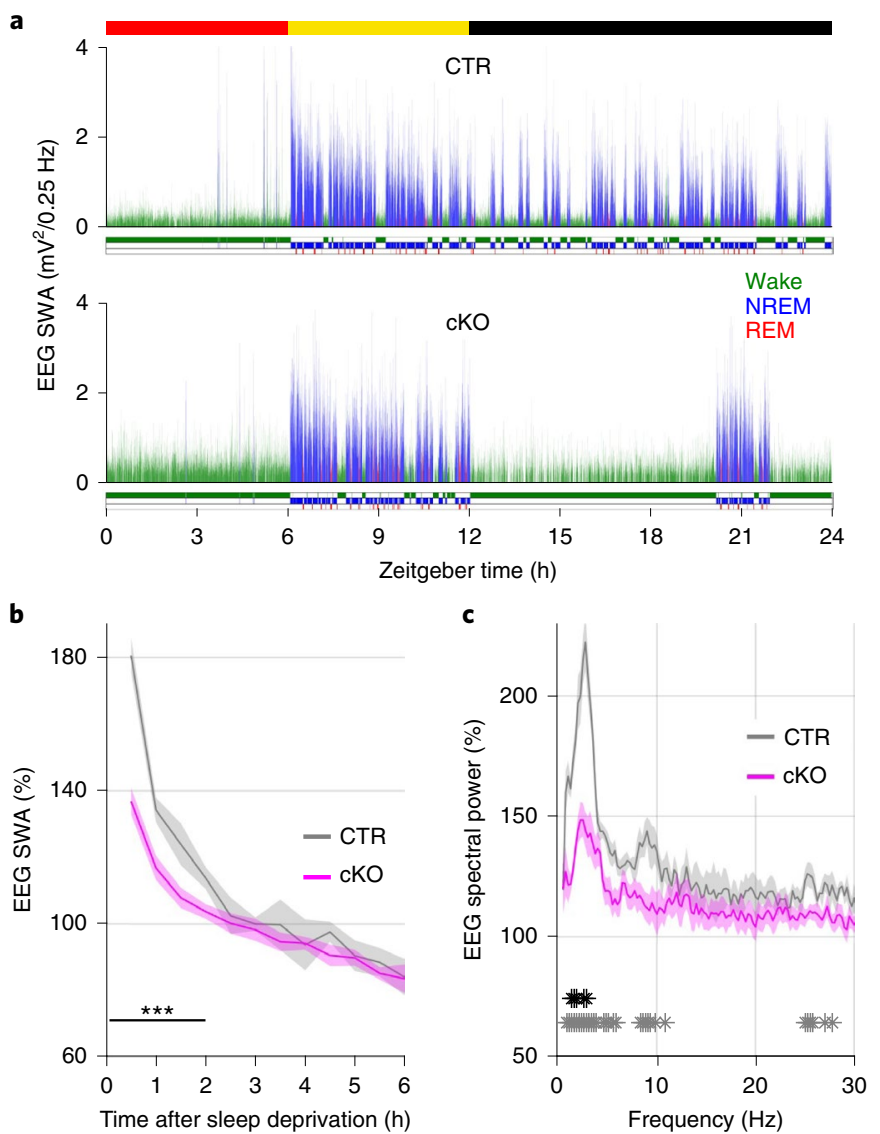

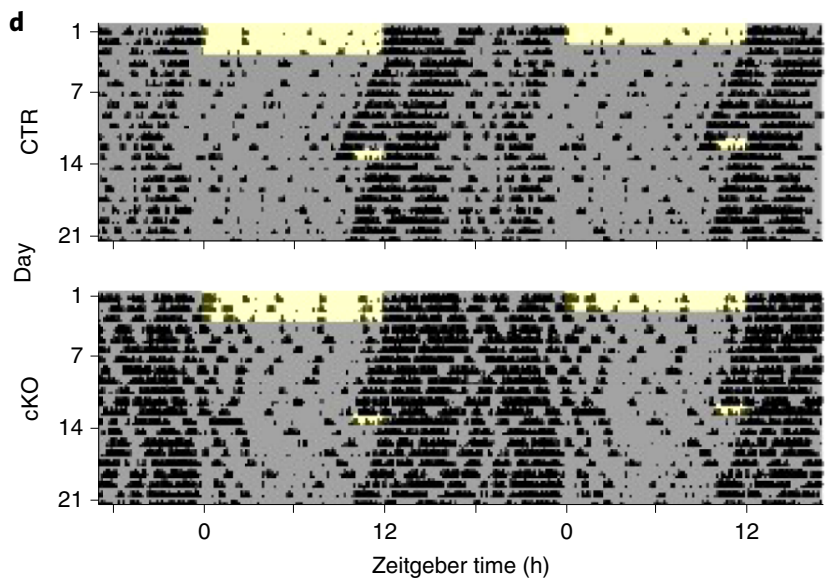

e
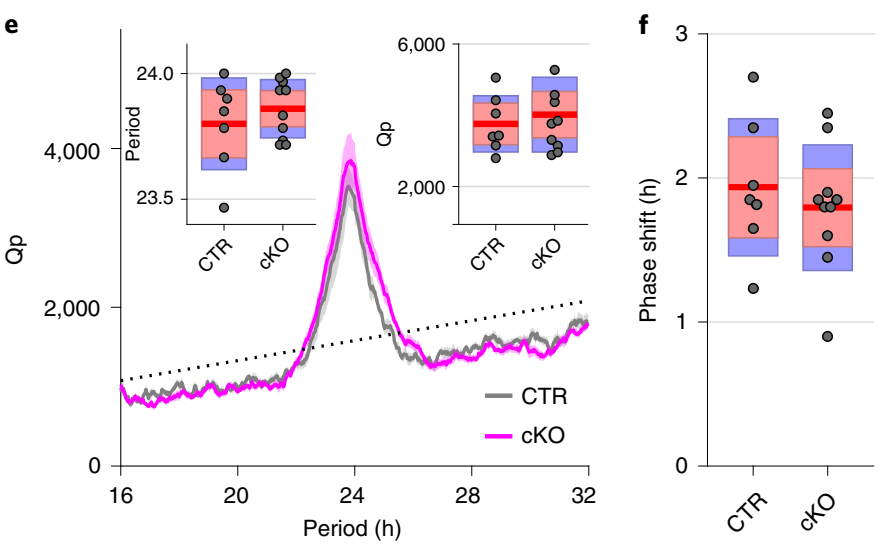

Fig. 3 | Selective cortical SNAP25 ablation alters homeostatic but not circadian sleep regulation. a-c, Homeostatic response to 6-h sleep deprivation at light onset based on EEG spectral analysis. $\mathbf{a}$, Hypnogram and EEG SWA (0.5-4.0 Hz, 4-s epochs) of one representative mouse from each genotype (red, yellow and black bars indicate sleep deprivation, light period and dark period, respectively). $\mathbf{b}$, Time course of frontal EEG SWA during NREM sleep after sleep deprivation relative to baseline average (genotype $\times$ time interaction: $F(11,121)=7.561, P<0.001$, mixed ANOVA). Note the reduced SWA in cKO compared to CTR mice during the first $2 \mathrm{~h}$ after sleep deprivation. c, NREM sleep spectral power in the frontal derivation during the first 30 min after sleep deprivation relative to baseline NREM average (genotype $\times$ frequency interaction: $F(118,1298)=4.068, P<0.001$, mixed ANOVA). Asterisks indicate frequency bins with significant differences in post hoc comparison before (gray) and after (black) Bonferroni adjustment of $\alpha$. d-f, Circadian phenotyping based on passive infrared recordings. d, Representative actograms of one mouse from each genotype. Days 1-3: 12:12-h light:dark cycle. From day 4: constant darkness protocol. Day 13: phase-delaying 2-h light pulse. e, Average chi-squared periodogram. Insets show period (h) and periodogram power (Qp).f, Phase shift in activity onset the day after the light pulse. No significant differences were observed in any of the assessed circadian characteristics. $n=5$ CTR and $n=8 \mathrm{cKO}$ for EEG spectral analysis (b and $\mathbf{c}$ ), $n=7$ CTR and $n=10 \mathrm{cKO}$ for passive infrared recording analysis (e and $\mathbf{f}$ ). Black asterisks indicate post hoc contrasts with significant differences $\left({ }^{\star} P<0.05,{ }^{\star \star} P<0.01,{ }^{\star \star \star} P<0.001\right.$ ). Data in $\mathbf{b}, \mathbf{c}$ and $\mathbf{e}$ are presented as the mean \pm s.e.m. (shaded areas). Data in $\mathbf{e}$ and $\mathbf{f}$ represent grouped data including the group mean (red line), 95\% confidence interval (pink box) and one standard deviation (blue box) with individual data points overlaid. See Supplementary Table 1 for detailed results.

Fig. 6), which is the mouse's circadian active period but also the time of day when the homeostatic sleep drive typically builds up to high levels as a result of prolonged wakefulness ${ }^{21}$. This raises the question whether the increase in wakefulness is due to changes in the homeostatic or circadian process of sleep regulation.

To assess the buildup of the homeostatic sleep drive during spontaneous wakefulness, we compared the levels of EEG SWA during NREM sleep preceding and following individual wake episodes. As expected, a positive correlation was observed in both genotypes, whereby longer spontaneous wake episodes were followed by proportionally higher levels of SWA during NREM sleep. However, the increase of SWA relative to the duration of wake episodes was smaller in cKO mice compared to that in controls (Fig. $2 \mathrm{f}$ and Supplementary Table 1), indicating that the relationship between sleep-wake history and the levels of SWA might be altered in cortical SNAP25-ablated animals.

An established approach to investigate the dynamics of sleep homeostasis is sleep deprivation, which is most commonly performed starting at light onset, when mice in laboratory conditions usually sleep $^{3,21}$. Typically, sleep deprivation leads to a small increase in sleep amount, especially NREM sleep, and a strong increase in sleep intensity, reflected in SWA during NREM sleep ${ }^{21,22}$. However, we observed a striking difference in this homeostatic rebound between genotypes (Fig. 3a-c). Although many cortical SNAP25-ablated animals were spontaneously awake prior to the onset of sleep deprivation (Supplementary Fig. 4a), they did not spend more time asleep after sleep deprivation than controls when sleep deprivation was performed during the first half of the light period (Extended Data Fig. 7). When the time window of the sleep deprivation was shifted to the second half of the light period, the relative amount of sleep over the following $24 \mathrm{~h}$ was reduced in cortical SNAP25-ablated mice compared to controls (Supplementary Fig. 4b). Moreover, cKO mice presented a marked attenuation of the initial increase of EEG SWA during NREM sleep after sleep deprivation (relative SWA: cortical SNAP25-ablated animals, $136.77 \% \pm 3.98 \%$; controls, $180.57 \% \pm 5.13 \%$; $t(11)=6.78$, $P<0.001, d=-3.87$; Fig. $3 \mathrm{~b}, \mathrm{c})$. Consistent with the notion of a 
frontal predominance of the homeostatic response to sleep deprivation $^{23}$, the genotype difference in SWA rebound was observed in the frontal EEG and LFPs, but not in the occipital EEG derivation (Extended Data Fig. 8). We also observed that the increase in EEG theta activity, a measure of 'wake intensity', was attenuated in cKO mice during sleep deprivation in both the frontal and occipital EEG derivation (Extended Data Fig. 9).

Because the circadian and the homeostatic processes interact in the regulation of sleep and wakefulness, we next assessed whether clock function was altered in cKO mice, using a separate cohort of animals. A standard approach in circadian phenotyping is to continuously monitor locomotor activity using a passive infrared recording system under a 12:12-h light:dark cycle followed by a release into constant darkness and exposure to a phase-advancing light pulse during the early subjective night (circadian time $\sim 13.5$ h; Fig. $3 \mathrm{~d}$ ) ${ }^{24}$. We replicated the approximately 3 -h difference in the amount of wakefulness in this new cohort of animals (cortical SNAP25-ablated mice: $11.79 \pm 0.29 \mathrm{~h}$, controls: $9.10 \pm 0.51 \mathrm{~h} ; t(15)=4.957, P<0.001$, $d=2.35$ ) and observed that the sleep phenotype remained stable in constant darkness (Extended Data Fig. 10). Importantly, cortical SNAP25-ablated animals remained rhythmic in the absence of light (Fig. 3d). The free-running period was slightly shorter than $24 \mathrm{~h}$ and nearly identical in both genotypes (cortical SNAP25-ablated mice: $23.86 \pm 0.04 \mathrm{~h}$, controls: $23.80 \pm 0.07 \mathrm{~h} ; t(15)=0.833, P=0.42$, $d=0.39$ ), and the chi-squared periodogram analysis did not reveal any difference in circadian amplitude (Fig. 3e). Finally, irrespective of genotype, the light pulse evoked a consistent phase delay, which was comparable between genotypes (Fig. 3f). Taken together, our data demonstrate that cortical SNAP25 ablation leads to a diminished homeostatic sleep drive without affecting circadian regulation of sleep.

The regulation of sleep in mammals is only partially understood because it is unclear where and in what form the need to sleep is encoded, and how it is translated into an adequate compensatory response $\mathrm{e}^{25}$. Our study reveals an essential role for the cortex in sleep-wake regulation. We show that cortical structures actively contribute to sleep homeostasis and the global control of vigilance states. This supports the hypothesis that brain structures fundamentally involved in sleep regulation extend far beyond the traditionally considered subcortical circuitry ${ }^{2,7,26,27}$. The next step will be to understand how neocortical and archicortical neurons interact with established circuits of sleep-wake control. It was recently reported that axons of layer 5 pyramidal neurons in prefrontal cortex produce an axonal terminal field in the lateral hypothalamus ${ }^{28}$. Consistent with this observation, we found dense fine Cre-positive fibers surrounding cell bodies in the lateral hypothalamus (Extended Data Fig. 4). Long-range projections from prefrontal cortex, which is known to be highly sensitive to homeostatic sleep pressure ${ }^{23}$ and a hub for the generation of slow waves during NREM sleep ${ }^{29}$, might represent a direct pathway through which the neocortex could modulate the control of vigilance states by the lateral hypothalamus. However, layer 5 pyramidal neurons also project to thalamic nuclei, and could influence sleep regulation through corticothalamic loops $\mathrm{s}^{30,31}$. Given that layer 5 pyramidal neurons have a wide range of efferent connections to target structures involved in sleepwake control, a systematic and unbiased dissection of the relevant circuits is warranted. Furthermore, a potential involvement of other neocortical cell types and of archicortical dentate gyrus cells of the hippocampus should be considered. Given the critical place of the hippocampus in brain-wide circuitry involved in memory and temporal processing, one could speculate that this structure may have a so far unrecognized role in encoding time spent awake or asleep. Targeted manipulations of the hippocampus and its neocortical projections could shed further light on the relationship between REM and NREM oscillations and their role in global sleep-wake regulation and function ${ }^{32}$.
Our results support the possibility that cortical structures generate sleep drive locally, in an activity-dependent fashion ${ }^{7}$, and raise the question of which mechanisms the cortex could use to generate, sense and/or integrate signals of sleep need. Extracellular signals may be found in molecular regulators of inflammation and plasticity $^{7}$, or adenosine levels regulated through neural-glial interactions. Intracellular processes reflecting wake-dependent increases in sleep need, conserved both in mammalian and nonmammalian species, may represent changes in the synaptic phosphoproteome, endoplasmic reticulum stress or redox homeostasis. Arguably, such local signals must be integrated to elicit a global homeostatic response $^{33}$, reflected in an occurrence of intense sleep, characterized by elevated cortical SWA and increased sleep propensity. We propose that the wide connectivity of layer 5 pyramidal neurons to other parts of cortex, thalamus and sleep-wake regulating nuclei in hypothalamus ${ }^{28}$ and brain stem ${ }^{16}$, places this neuronal population in an ideal position not only to generate SWA, but also to sense and integrate the signals related to sleep need, and ultimately broadcast the information to the subcortical circuitry responsible for sleepwake switching ${ }^{1}$.

\section{Online content}

Any methods, additional references, Nature Research reporting summaries, source data, extended data, supplementary information, acknowledgements, peer review information; details of author contributions and competing interests; and statements of data and code availability are available at https://doi.org/10.1038/ s41593-021-00894-6.

Received: 17 April 2020; Accepted: 23 June 2021; Published online: 2 August 2021

\section{References}

1. Saper, C. B. \& Fuller, P. M. Wake-sleep circuitry: an overview. Curr. Opin. Neurobiol. 44, 186-192 (2017).

2. Liu, D. \& Dan, Y. A motor theory of sleep-wake control: arousal-action circuit. Annu. Rev. Neurosci. 42, 27-46 (2019).

3. Vyazovskiy, V. V. et al. Cortical firing and sleep homeostasis. Neuron 63, 865-878 (2009).

4. Borbély, A. A., Daan, S., Wirz-Justice, A. \& Deboer, T. The two-process model of sleep regulation: a reappraisal. J. Sleep. Res. 25, 131-143 (2016).

5. Huber, R., Felice Ghilardi, M., Massimini, M. \& Tononi, G. Local sleep and learning. Nature 430, 78-81 (2004).

6. Vyazovskiy, V. V. et al. Local sleep in awake rats. Nature $\mathbf{4 7 2}$ 443-447 (2011).

7. Krueger, J., Nguyen, J. T., Dykstra-Aiello, C. J. \& Taishi, P. Local sleep. Sleep. Med. Rev. 43, 14-21 (2019).

8. Krone, L. B. \& Vyazovskiy, V. V. Unresponsive or just asleep? do local slow waves in the perilesional cortex have a function? Brain 143 , 3513-3515 (2020)

9. Beltramo, R. et al. Layer-specific excitatory circuits differentially control recurrent network dynamics in the neocortex. Nat. Neurosci. 16, 227-234 (2013)

10. Chauvette, S., Volgushev, M. \& Timofeev, I. Origin of active states in local neocortical networks during slow sleep oscillation. Cereb. Cortex 20, 2660-2674 (2010).

11. Sanchez-Vives, M. V. \& McCormick, D. A. Cellular and network mechanisms of rhytmic recurrent activity in neocortex. Nat. Neurosci. 3, 1027-1034 (2000).

12. Buzsáki, G. Theta oscillations in the hippocampus. Neuron 33, 325-340 (2002).

13. Boyce, R., Glasgow, S. D., Williams, S. \& Adamantidis, A. Causal evidence for the role of REM sleep theta rhythm in contextual memory consolidation. Science 352, 812-816 (2016).

14. Sanchez-Vives, M. V., McCormick, D. A., Sanchez-Vives, M. V. \& McCormick, D. A. Cellular and network mechanisms of rhythmic recurrent activity in neocortex. Nat. Neurosci. 3, 1027-1034 (2000).

15. Hoerder-Suabedissen, A. et al. Cell-specific loss of SNAP25 from cortical projection neurons allows normal development but causes subsequent neurodegeneration. Cereb. Cortex 29, 2148-2159 (2019).

16. Gerfen, C. R., Paletzki, R. \& Heintz, N. GENSAT BAC cre-recombinase driver lines to study the functional organization of cerebral cortical and basal ganglia circuits. Neuron 80, 1368-1383 (2013). 
17. Lefort, S., Tomm, C., Floyd Sarria, J.-C. \& Petersen, C. C. H. The excitatory neuronal network of the $\mathrm{C} 2$ barrel column in mouse primary somatosensory cortex. Neuron 61, 301-316 (2009).

18. Vecchia, D. et al. Temporal sharpening of sensory responses by layer $\mathrm{V}$ in the mouse primary somatosensory cortex. Curr. Biol. 30, 1589-1599 (2020).

19. Washbourne, P. et al. Genetic ablation of the t-SNARE SNAP-25 distinguishes mechanisms of neuroexocytosis. Nat. Neurosci. 5, 19-26 (2002).

20. Funk, C. M., Honjoh, S., Rodriguez, A. V., Cirelli, C. \& Tononi, G. Local slow waves in superficial layers of primary cortical areas during REM sleep. Curr. Biol. 26, 396-403 (2016).

21. Huber, R., Deboer, T. \& Tobler, I. Effects of sleep deprivation on sleep and sleep EEG in three mouse strains: empirical data and simulations. Brain Res. 857, 8-19 (2000).

22. Franken, P., Dijk, D. J., Tobler, I. \& Borbely, A. A. Sleep deprivation in rats: effects on EEG power spectra, vigilance states, and cortical temperature. Am. J. Physiol. 261, R198-R208 (1991).

23. Huber, R., Deboer, T. \& Tobler, I. Topography of EEG dynamics after sleep deprivation in mice. J. Neurophysiol. 84, 1888-1893 (2000).

24. Albrecht, U. \& Foster, R. G. Placing ocular mutants into a functional context: a chronobiological approach. Methods 28, 465-477 (2002).

25. Frank, M. G. \& Heller, H. C. The function(s) of sleep. Handb. Exp. Pharmacol. 253, 3-34 (2019).

26. Morairty, S. R. et al. A role for cortical nNOS/NK1 neurons in coupling homeostatic sleep drive to EEG slow wave activity. Proc. Natl Acad. Sci. USA 110, 20272-20277 (2013).
27. Tossell, K. et al. Sleep deprivation triggers somatostatin neurons in prefrontal cortex to initiate nesting and sleep via the preoptic and lateral hypothalamus. Preprint at bioRxiv https://doi.org/10.1101/2020.07.01.179671 (2020).

28. Prasad, J. A., Carroll, B. J. \& Sherman, S. M. Layer 5 corticofugal projections from diverse cortical areas: variations on a pattern of thalamic and extrathalamic targets. J. Neurosci. 40, 5785-5796 (2020).

29. Massimini, M., Huber, R., Ferrarelli, F., Hill, S. \& Tononi, G. The sleep slow oscillation as a traveling wave. J. Neurosci. 24, 6862-6870 (2004).

30. Krone, L. et al. Top-down control of arousal and sleep: fundamentals and clinical implications. Sleep. Med. Rev. 31, 17-24 (2017).

31. Gent, T. C., Bandarabadi, M., Herrera, C. G. \& Adamantidis, A. Thalamic dual control of sleep and wakefulness. Nat. Neurosci. 21, 974-984 (2018).

32. Hayashi, Y. et al. Cells of a common developmental origin regulate REM/non-REM sleep and wakefulness in mice. Science 350, 957-961 (2015).

33. Thomas, C. W., Guillaumin, M. C. C., McKillop, L. E., Achermann, P. \& Vyazovskiy, V. V. Global sleep homeostasis reflects temporally and spatially integrated local cortical neuronal activity. Elife 9, e54148 (2020).

Publisher's note Springer Nature remains neutral with regard to jurisdictional claims in published maps and institutional affiliations.

(C) The Author(s), under exclusive licence to Springer Nature America, Inc. 2021 


\section{Methods}

Animals. Rbp4-Cre;Ai14;Snap2 $5^{\mathrm{A} / \mathrm{l}}$ is a triple transgenic mouse line, which was designed as a model for functional silencing of cortical layer 5 pyramidal and dentate gyrus granule cells. Snap $25^{\mathrm{t} / \mathrm{ll}}$ is a transgene, with lox-P sites flanking the alternatively spliced exons 5a and 5b of the t-SNARE gene Snap25. Cre-dependent excision of exon $5 \mathrm{a} / 5 \mathrm{~b}$ leads to a reduced length gene transcript and non-detectable levels of SNAP25 protein, and cessation of $\mathrm{Ca}^{2+}$-dependent evoked synaptic vesicle release ${ }^{15}$. Because this chronic t-SNARE disruption allows cortex-wide silencing of selected cell types while avoiding a mechanical manipulation of cortex, which is known to affect the expression of SWA ${ }^{34}$, we opted for this silencing method. In addition, our choice of this mouse model was guided by previous neurodevelopmental studies on the effects of disrupted evoked neurotransmitter release through ablation of SNARE proteins ${ }^{19,35,36}$, as well as by neuroanatomical work conducted in the Rbp4-Cre;Ai14;Snap $25^{\mathrm{f} / \mathrm{fl}}$ mouse line ${ }^{15}$. These studies consistently report that brain development, in particular cortical layering and axonal pathfinding, is unaffected by ablation of SNAP25 (refs. ${ }^{15,19}$ ). Moreover, as sleep homeostasis occurs on a timescale of minutes to hours, is most likely a distributed process ${ }^{33,37}$, and it is not yet known whether specific areas of the cortex are more involved than others, we considered the Rbp4-Cre;Ai14;Snap25 $5^{\mathrm{f} / \mathrm{ll}}$ mouse model most suitable for the aims of this study ${ }^{16}$. Spontaneous behavior appeared indistinguishable between genotypes, but cKO mice have a lower body weight compared to Cre-negative controls (cortical SNAP25-ablated mice: $21.3 \pm 0.6 \mathrm{~g}$, controls: $24.4 \pm 0.6 \mathrm{~g} ; t(18)=2.94, P=0.009$ ), as reported previously ${ }^{15}$.

Chronic electrophysiological recordings. EEG/EMG implants were performed in 7 wild-type C57BL/ 6 mice ( $125 \pm 8 \mathrm{~d}$ old at baseline recording), 12 Rbp4-Cre;Ai14;Snap $25^{\mathrm{f} / \mathrm{fl}}$ (cKO) mice ( $90 \pm 5 \mathrm{~d}$ old at baseline recording) and 8 Cre-negative (control) littermates $(85 \pm 4 \mathrm{~d}$ old at baseline recording) under isoflurane anesthesia as described previously ${ }^{38}$. For analysis of sleep architecture based on EEG/EMG recordings, $9 \mathrm{cKO}$ and 6 control mice were included. EEG analysis of frontal and occipital spectra was conducted in $8 \mathrm{cKO}$ and $5 \mathrm{control}$ mice. Laminar LFP and MUA could be obtained across cortical layers in primary motor cortex (+1.1 $\mathrm{mm} \mathrm{AP} \mathrm{(anterior),}-1.75 \mathrm{~mm} \mathrm{ML} \mathrm{(left),} \mathrm{tilt}-15^{\circ}$ (left)) from 7 wild-type, $5 \mathrm{cKO}$ and 5 control mice. All laminar recordings were performed using 16-channel silicon probes (NeuroNexus Technologies; A1 $\times 16-3 \mathrm{~mm}-100$ 703-Z16) with a spacing of $100 \mu \mathrm{m}$ between individual channels. All experiments were conducted in young adult male mice, because estrous cycle, development and aging affect sleep.

All mice implanted for electrophysiological recordings were housed individually in open cages before surgery and in individually ventilated cages during a recovery period of about 1 week after surgery. For sleep recordings, mice were transferred to separate custom-made Plexiglas cages $(20.3 \times 32 \times 35 \mathrm{~cm})$, which were placed in sound-attenuated and light-controlled Faraday chambers (Campden Instruments), with each chamber fitting two cages. Animals were allowed free access to food pellets and water at all times and underwent daily health inspection. A 12:12-h light:dark cycle (lights on at 09:00, light levels 120-180 lux) was implemented, temperature maintained at around $22 \pm 2{ }^{\circ} \mathrm{C}$, and humidity kept around $50 \% \pm 20 \%$

After an acclimatization period of at least $3 \mathrm{~d}$ during which animals were habituated to the tethered recording conditions, a 24-h period of continuous recording starting at light onset was performed on a designated baseline day. On the subsequent day, all animals were sleep deprived for $6 \mathrm{~h}$ starting at light onset. Sleep deprivation was performed during the circadian period when mice are typically asleep and thus the homeostatic response to sleep loss can be most reliably elicited ${ }^{21}$. At light onset, recording chambers were opened, the nesting material removed and novel objects placed into the mouse cages to encourage exploratory behavior. Experimenters continuously observed the mice and exchanged the provided objects for new objects when mice stopped exploring. At the end of the 6-h sleep deprivation, all objects were removed, the nesting material returned, and the recording chambers closed. Sleep deprivation was successful in both genotypes, as only a minimal amount of time (cKO, $1.84 \% \pm 0.75 \%$; control, $1.39 \% \pm 0.32 \%$; $P=0.86$, Mann-Whitney $U$ test) was spent asleep during the 6 -h interval when the mice were kept awake by providing novel objects.

The electrophysiological signals revealed typical signatures of wakefulness and sleep states in both genotypes. As expected, the laminar profile of LFPs and MUAs revealed generally activated patterns during waking and REM sleep. Correspondingly, during NREM sleep, we observed depth-positive LFP slow waves associated with a generalized suppression of spiking activity across cortical layers (Fig. 1c) ${ }^{20,39}$.

\section{Electrophysiological acquisition, data processing and sleep scoring.}

Electrophysiological in vivo recordings. Data were acquired using the 128-channel Neurophysiology Recording System (Tucker-Davis Technologies) and the electrophysiological recording software Synapse (Tucker-Davis Technologies), and saved on a local computer. EEG and EMG signals were continuously recorded, filtered between $0.1-100 \mathrm{~Hz}$, and stored at a sampling rate of $305 \mathrm{~Hz}$. Extracellular neuronal activity was continuously recorded at a sampling rate of $25 \mathrm{kHz}$ and filtered between $300 \mathrm{~Hz}-5 \mathrm{kHz}$. Whenever the recorded voltage in an individual laminar channel crossed a manually set threshold indicating putative neuronal firing (at least two standard deviations above noise level), 46 samples around the event ( $0.48 \mathrm{~ms}$ before, $1.36 \mathrm{~ms}$ after) were stored. Concomitantly with the spike acquisition, LFPs were continuously recorded from the same electrodes and processed with the abovementioned settings for EEG signals.

Offline signal processing. EEG, EMG and LFP signals were resampled at a sampling rate of $256 \mathrm{~Hz}$ using custom-made code in MATLAB (MathWorks, v2017a) and converted into European Data Format as previously described ${ }^{38}$. Spike waveforms were further processed using a custom-made MATLAB script and events with artifactual waveforms were excluded from further analysis of neuronal activity.

Scoring of vigilance states. The software SleepSign for Animals (v3.3.6.1602, SleepSign Kissei Comtec) was used for sleep scoring. EEG, EMG and LFP recordings were partitioned into epochs of $4 \mathrm{~s}$. Vigilance states were assigned manually to each recording epoch based on visual inspection of the frontal and occipital EEG derivations in conjunction with the EMG. Epochs with recording artifacts due to gross movements, chewing or external electrostatic noise were assigned to the respective vigilance state but not included in the electrophysiological analysis. Overall, $18.8 \% \pm 3.5 \%$ of wake, $0.7 \% \pm 0.4 \%$ of NREM and $0.9 \% \pm 0.4 \%$ of REM epochs contained artifactual EEG signals across all animals included in the EEG spectral analysis, with no significant difference between genotypes. EEG and LFP power spectra were computed using a fast Fourier transform routine (Hanning window) with a $0.25-\mathrm{Hz}$ resolution and exported in the frequency range between 0 and $30 \mathrm{~Hz}$ for spectral analysis.

Noninvasive measurement of home cage activity for circadian phenotyping. Circadian characteristics were assessed in a separate cohort of ten cKO $(84 \pm 1 \mathrm{~d}$ old at baseline recording) and seven control ( $83 \pm 1 \mathrm{~d}$ old at baseline recording) mice. These mice were housed individually and placed under a passive infrared motion detector that continuously recorded activity in time intervals of $100 \mathrm{~ms}$. Individual readings were pooled into 1-min bins for all analyses as it has previously been validated that periods of inactivity of $>40$ s provide a reliable measure of behaviorally defined sleep ${ }^{40}$. Mice were housed in a stable 12:12-h light:dark cycle for at least $7 \mathrm{~d}$ before being released into constant darkness. The circadian period and amplitude were assessed over an interval of 7-10 d in the constant dark condition by chi-squared periodogram analysis using Actogram $\mathrm{J}^{41}$. After at least $10 \mathrm{~d}$ in constant darkness, all mice were exposed to a 2 -h light pulse provided around circadian time 13.5. The resulting phase delay of the circadian rhythm was quantified in all animals by extrapolating the onsets of activity to the day of the light pulse ${ }^{24}$.

Experimental design and statistical analyses. Sample sizes were initially based on previous studies ${ }^{6,21}$. Power calculations were performed after acquisition of pilot data for the Wellcome Trust grant proposal 203971/Z/16/A indicated an effect size of $d=2.75$ for the main outcome parameter NREM sleep time over $24 \mathrm{~h}$. Applying an intended power of 0.9 and an error probability alpha level of 0.01 , the power calculations confirmed the initial sample size estimate of $n=8$ animals per genotype considering an attrition rate of $25 \%$. Animals were retrieved from the breeder colony upon availability. Based on the breeding scheme, we expected that male Rbp4-Cre;Ai14;Snap25 $5^{\mathrm{t} / \mathrm{l} l}$ mice only represent on average $1 / 8$ of the animals in each litter. As soon as animals of the desired genotype were available, they were included in the study and the recording capacities were filled with randomly selected Cre-negative controls from the colony, preferably littermates. Data were analyzed using MATLAB (vR2017b; MathWorks), JMP (v7.0; SAS Institute) and SPSS Statistics for Windows (v25.0; IBM). Reported averages are the mean \pm s.e.m. ANOVAs were performed as described in work by Howell and Lacroix ${ }^{42}$. To examine potential differences in firing dynamics between superficial versus deep cortical layers in wild-type animals, repeated-measures ANOVAs were conducted with cortical layers (layer 2/3 versus layer 5 ) and time bins as within-subject factors. To examine potential differences between genotypes, split-plot ANOVAs were conducted; genotype (control versus cKO animals) was entered as a between-subject factor, whereas time bins, EEG spectral bins and vigilance states (wake, NREM and REM) were entered as within-subject factors. A value of $\alpha=0.05$ was adopted for main effects and interactions. Significant two-way interaction terms were followed up with Bonferroni-adjusted post hoc comparisons with $\alpha_{\text {adjusted }}=0.05 / k$, where $k$ represents the total number of pairwise comparisons made $^{43}$. In ANOVAs with multiple time points, the total number of post hoc comparisons was minimized in each case, and hence controlling for the increase in family-wise error rate, by pooling across multiple time bins (for example, 0-10 ms, $11-20 \mathrm{~ms}$ and $21-30 \mathrm{~ms}$ in Fig. 1g). For spectral analysis, EEG/LFP power spectra of individual animals were log transformed before hypothesis testing. Bonferroni correction was not applied for post hoc comparison of spectral data because ANOVAs consisting of 119 EEG spectral bins would be too conservative and reduce statistical power ${ }^{44}$; thus, $\alpha$ was kept at 0.05 in these cases. In the summary of statistical methods and results (Supplementary Table 1), we report frequency bins with significant differences in post hoc comparison before $\left(\alpha_{\text {uncorrected }}\right)$ and after $\left(\alpha_{\text {corrected }}\right)$ Bonferroni adjustment of $\alpha$. In all figures illustrating EEG or LFP spectrograms, frequency bins for which the resulting post hoc comparison was significant after Bonferroni adjustment are highlighted with 
black asterisks; bins only significant before Bonferroni adjustment are highlighted with gray asterisks to indicate areas of interest, bearing in mind that no correction for multiple comparisons was applied. In the circadian analysis, a multivariate ANOVA was conducted to examine if the linear combination of multiple circadian response variables (period length, periodogram power and phase shift) differed between genotypes ${ }^{45}$. Data distribution was assessed graphically and formally tested if normality and equal variances could not be assumed. Greenhouse-Geisser correction was used when the assumption of sphericity was violated (Mauchly's tes of sphericity, $P<0.05)$. Mann-Whitney $U$ tests were performed for main analyses instead of one-way ANOVAs if the assumption of normality was violated (Shapiro-Wilk test of normality, $P<0.05$ ). The statistical methods and results from each individual analysis are listed in Supplementary Table 1. In all figures, significance levels are indicated with black asterisks: ${ }^{\star} 0.05 \geq P>0.01$; ${ }^{* *} 0.01 \geq P>0.001 ;{ }^{* *} 0.001 \geq P$. Gray asterisks indicate post hoc comparisons with $P<0.05$, which did not reach significance after Bonferroni correction for multiple comparisons. Grouped data of durations spent in specific vigilance states show the group mean (red line), 95\% confidence interval (pink box) and one standard deviation (blue box) with individual data points overlaid; these plots were generated using the MATLAB functions notBoxPlot (retrieved from GitHub at https://www.github.com/raacampbell/notBoxPlot/ on 15 June 2019) and RGB (retrieved from MATLAB Central File Exchange at https://www.mathworks.com/ matlabcentral/fileexchange/24497-rgb-triple-of-color-name-version-2/ on 15 June 2019). For key analyses reported in the main text, the effect sizes are reported as Cohen's $d$ calculated using the MATLAB function computeCohen_d $(\mathrm{x} 1, \mathrm{x} 2$, varargin; retrieved from MATLAB Central File Exchange at https://www.mathworks.com/matlabcentral/fileexchange/62957-computecohen d-x1-x2-varargin/ on 4 October 2020).

Electrophysiological criteria for analysis of vigilance state episodes and OFF periods. For analyses of mean and maximum duration of sustained wake episodes in the EEG dataset (Fig. 2e), we included wake episodes, which were at least 1 -min long allowing brief intrusions of sleep of 1 min or less. For the analysis of mean duration of NREM episodes, we included NREM episodes, which were at least 1-min long allowing brief intrusions of REM sleep or brief awakenings of 1 min or less (Extended Data Fig. 7). To investigate the change in NREM SWA across prolonged wake episodes under undisturbed conditions, we used the EEG dataset from the baseline day and included consolidated periods of waking lasting at least $15 \mathrm{~min}$, whereby short episodes of sleep $<1 \mathrm{~min}$ were not considered as interruptions. We then performed analyses of NREM sleep SWA in the 15-min time window immediately preceding and following prolonged ( $>15 \mathrm{~min})$ wake episodes, if both time windows included at least $10 \mathrm{~min}$ of artifact-free NREM sleep and no more than $3 \mathrm{~min}$ of wakefulness (Fig. 2f). Population OFF periods were defined as periods of total neuronal silence across all electrodes, which lasted at least $50 \mathrm{~ms}$ and no more than $4,000 \mathrm{~ms}$. Subsequently, the top $20 \%$ longest OFF periods were included for final analyses (Fig. 1g,h and Extended Data Fig. 1). The latency to the first spike after the population OFF-ON transition was calculated separately for MUA recorded in layer $2 / 3$ and layer 5 . Only $O N$ periods with at least one spike in each of the layers occurring within the first $200 \mathrm{~ms}$ were included in this analysis (Extended Data Fig. 1).

Histological assessment of laminar probe depth. The tips of laminar implants were stained before surgery with the orange/red fluorescence membrane stain DiI (Thermo Fisher Scientific) by immersion of the electrode shank into a $20 \mathrm{mg}$ $\mathrm{ml}^{-1}$ solution $(50 / 50 \%$ acetone/methanol) for later histological assessment of the electrode position ${ }^{46}$. After completion of the experiments, microlesions of selected channels on the laminar probe were performed under terminal pentobarbital anesthesia using the electroplating device NanoZ (White Matter) applying $10 \mathrm{~mA}$ of direct current for $25 \mathrm{~s}$ to each respective channel. Immediately following microlesioning, mice were perfused with $0.1 \mathrm{M}$ PBS $(0.9 \%)$ followed by $4 \%$ paraformaldehyde in PBS for tissue preservation. A vibrating microtome (Leica VT1000S) was used to section the brains into $50-\mu \mathrm{m}$ coronal slices. Fluorescence staining was performed with DAPI. After fluorescence microscopy, implantation sites were mapped using a mouse brain atlas ${ }^{47}$ and the depth of the laminar implant was assessed measuring the distance between cortical surface and the electrical current induced tissue microlesions. The histological assessment of the insertion tract was performed in all animals, which were implanted with laminar probes. Structural integrity of cortex and the position of the laminar implant similar to those shown in the representative examples (Fig. 1d and Supplementary Fig. 2b) were found in each individual animal included in the electrophysiological analysis of laminar data. ImageJ (v1.52a) was used to merge fluorescence images and add scale bars ${ }^{48}$. All figures were created using Inkscape (v1.0.2, Inkscape Project 2020; https://inkscape.org)

Histological assessment of Cre expression in cortex, dentate gyrus and hypothalamus. Coronal brain sections from six Rbp4-Cre;Ai14;Snap25 $5^{\mathrm{fl} /+}$ mice were prepared using the same procedure as outlined above for the histological assessment of the laminar probe depth. Laser scanning confocal microscope images were acquired (Zeiss LSM710) and image stacks created to assess the cellular morphology across the $z$ plane of the brain section. The cytoplasmic
tdTomato expressed from the Ai14 reporter gene allows reliable identification of Cre-positive neurons based on native tdTomato fluorescence ${ }^{15,49}$. The native fluorescence is detectable even in thin neurites and synapses, which can be used to visualize the dendritic and axonal morphology of labeled neurons. The histological assessment of Cre expression was performed in six Rbp4-Cre;Ai14;Snap25 $5^{\mathrm{t} /+}$ mice, and the results across all animals were similar to those presented in the representative images.

Immunohistochemistry to determine lateral hypothalamic cell identity. To determine whether Cre-positive neurons in the lateral hypothalamus express melanin-concentrating hormone $(\mathrm{MCH})$ or orexin/hypocretin (Hcrt), coronal sections from three Rbp4-Cre;Ai14 mice were prepared as described above. Before counterstaining with DAPI, the sections were stained with rabbit anti-MCH antibody (1:2,000 dilution; H-070-47, Phoenix Pharmaceuticals) or rabbit anti-Hcrt antibody (1:500 dilution; kind gift from A. van den Pol, Yale University). Briefly, sections were incubated in blocking solution (3\% or $10 \%$ donkey serum (Hcrt or $\mathrm{MCH}$, respectively) and $0.3 \%$ Triton-X100 in $0.1 \mathrm{M}$ PBS) for $1 \mathrm{~h}$ before incubation with the primary antibody in blocking solution at $4{ }^{\circ} \mathrm{C}$ overnight $(\mathrm{MCH})$ or for $72 \mathrm{~h}$ (Hcrt). The primary antibody was revealed by incubating with donkey anti-rabbit Alexa Fluor 488 (1:500 dilution) antibody in blocking solution for $2 \mathrm{~h}$ at room temperature. Subsequently, epifluorescence and confocal imaging was performed as described above.

Ethical approval. All experiments were performed in accordance with the United Kingdom Animal Scientific Procedures Act 1986 under personal and project licenses granted by the United Kingdom Home Office. Ethical approval was provided by the Ethical Review Panel at the University of Oxford. Animal holding and experimentation were located at the Biomedical Sciences Building and the Behavioural Neuroscience Unit, University of Oxford.

Reporting summary. Further information on experimental design and reagents is available in the nature Research Life Sciences Reporting Summary linked to this paper.

\section{Data availability}

A sample dataset with spectral data and sleep scoring results used to generate key analyses presented in this paper is available on Figshare (https://doi.org/10.6084/ m9.figshare.14737569). Source data are provided with this paper. Raw data from electrophysiological and passive infrared recordings are available from the corresponding authors upon reasonable request.

\section{Code availability}

Custom-made MATLAB code for key analyses is deposited on Figshare (https:// doi.org/10.6084/m9.figshare.14737578). Code used for additional analyses is available from the corresponding authors upon reasonable request.

\section{References}

34. Sheroziya, M. \& Timofeev, I. Global intracellular slow-wave dynamics of the thalamocortical system. J. Neurosci. 34, 8875-8893 (2014).

35 . Verhage, M. et al. Synaptic assembly of the brain in the absence of neurotransmitter secretion. Science 287, 864-869 (2000).

36. Molnár, Z. et al. Normal development of embryonic thalamocortical connectivity in the absence of evoked synaptic activity. J. Neurosci. 22, 10313-10323 (2002)

37. Krueger, J. \& Obál, F. A neuronal group theory of sleep function. J. Sleep. Res. 2, 63-69 (1993).

38. McKillop, L. E. et al. Effects of aging on cortical neural dynamics and local sleep homeostasis in mice. J. Neurosci. 38, 3911-3928 (2018).

39. Sakata, S. \& Harris, K. D. Laminar structure of spontaneous and sensory-evoked population activity in auditory cortex. Neuron $\mathbf{6 4}$, 404-418 (2009).

40. Brown, L., Hasan, S., Foster, R. G. \& Peirson, S. N. COMPASS: Continuous Open Mouse Phenotyping of Activity and Sleep Status. Wellcome Open Res. 1, 2 (2016).

41. Schmid, B., Helfrich-Förster, C. \& Yoshii, T. A new ImageJ plug-in 'ActogramJ' for chronobiological analyses. J. Biol. Rhythms 26, 464-467 (2011)

42. Howell, G. T. \& Lacroix, G. L. Decomposing interactions using GLM in combination with the COMPARE, LMATRIX and MMATRIX subcommands in SPSS. Tutorials Quant. Methods Psychol. 8, 1-22 (2012).

43. Howell, D. C. Statistical Methods for Psychology (Cengage Learning, 2012).

44. Achermann, P. \& Borbély, A. A. Coherence analysis of the human sleep electroencephalogram. Neuroscience 85, 1195-1208 (1998).

45. Tabachnick, B. G. \& Fidell, L. S. in International Encyclopedia of Statistical Science. (ed. Lovric, M.) 902-904 (Springer Berlin Heidelberg, 2011).

46. Magill, P. J. et al. Changes in functional connectivity within the rat striatopallidal axis during global brain activation in vivo. J. Neurosci. 26, 6318-6329 (2006) 
47. Paxinos, G. \& Franklin, K. B. J. The Mouse Brain in Stereotaxic Coordinates (Elsevier Academic Press, 2019).

48. Schindelin, J. et al. Fiji: an open-source platform for biological image analysis. Nat. Methods 9, 676-682 (2012).

49. Grant, E., Hoerder-Suabedissen, A. \& Molnar, Z. The regulation of corticofugal fiber targeting by retinal inputs. Cereb. Cortex 26, 1336-1348 (2016).

\section{Acknowledgements}

We thank T. Jahans-Price and X. Wang for help with establishing the microlesion protocol; M. Sabanovic for advice on statistics and figure production; K. Parley for support in the histological procedures; all members of the laboratory of V.V.V. for kind help with surgery assistance, animal care and sleep deprivation. We are very grateful to E. Mann, University of Oxford, for advice on the laminar analysis and to A. van den Pol, Yale University, for the generous gift of rabbit anti-Hcrt antibody. This work was supported by the Wellcome Trust PhD studentships 203971/Z/16/Z (to L.B.K.) and 109059/Z/15/Z (to C.B.-D.). T.Y. was supported by The Uehara Memorial Foundation Overseas Postdoctoral Fellowships, and The Naito Grant for Studying Overseas and a Wellcome Trust grant (106174/Z/14/Z). M.C.K. was supported by a Berrow Foundation Lord Florey Scholarship. M.C.C.G. was supported by a Biotechnology and Biological Sciences Research Council (BBSRC) DTP grant (BB/J014427/1) and by a Clarendon Scholarship (provided by the University of Oxford). V.v.d.V. and L.E.M. were supported by Novo Nordisk Postdoctoral Fellowships run in partnership with the University of Oxford. L.E.M. was also supported by a Sir Paul Nurse Junior Research Fellowship at Linacre College, Oxford. S.N.P. and S.K.E.T. are funded by the BBSRC (grant no. BB/S015817/1). The laboratory of Z.M. received funding from the UK Medical Research Council (G00900901), the Royal Society, St John's College Research Centre, the Anatomical Society and Einstein Stiftung. Z.M. is an Einstein Visiting Fellow at Charité-Universitätsmedizin Berlin (host B. Eickholt for 2020-2024), and lead researcher at Oxford Martin School, University of Oxford. This work was further supported by a Wellcome Trust Strategic Award (098461/Z/12/Z), a John Fell OUP Research Fund grant (131/032) and Medical Research Council (UK) grants MR/N026039/1 and MR/ $\mathrm{S} 01134 \mathrm{X} / 1$.

\section{Author contributions}

Z.M. and V.V.V. initiated and proposed the study with pilot experiments done by T.Y. L.B.K., T.Y., C.J.A., A.H.-S., Z.M. and V.V.V. designed the experiments. L.B.K., T.Y., M.C.C.G. and C.B.-D. conducted the electrophysiological experiments on the transgenic mouse model. L.B.K., C.B.-D. and M.C.K. conducted the electrophysiological experiments on wild-type mice. L.B.K., V.v.d.V., L.M.c.K., S.K.E.T. and S.N.P. conducted and analyzed the passive infrared recordings on the transgenic mouse model. L.B.K., M.C.K. and A.H.-S. performed the histology. A.H.-S. and Z.M. developed, validated and provided the transgenic mice. L.B.K., V.v.d.V., S.K.E.T. and V.V.V. analyzed the data. L.B.K. and V.V.V. wrote the manuscript. All authors discussed the results and commented on the manuscript.

\section{Competing interests}

The authors declare no competing interests.

\section{Additional information}

Extended data is available for this paper at https://doi.org/10.1038/s41593-021-00894-6.

Supplementary information The online version contains supplementary material available at https://doi.org/10.1038/s41593-021-00894-6.

Correspondence and requests for materials should be addressed to Z.M. or V.V.V.

Peer review information Nature Neuroscience thanks Marcos Frank, Akihiro Yamanaka, and the other, anonymous, reviewer(s) for their contribution to the peer review of this work.

Reprints and permissions information is available at www.nature.com/reprints. 
a

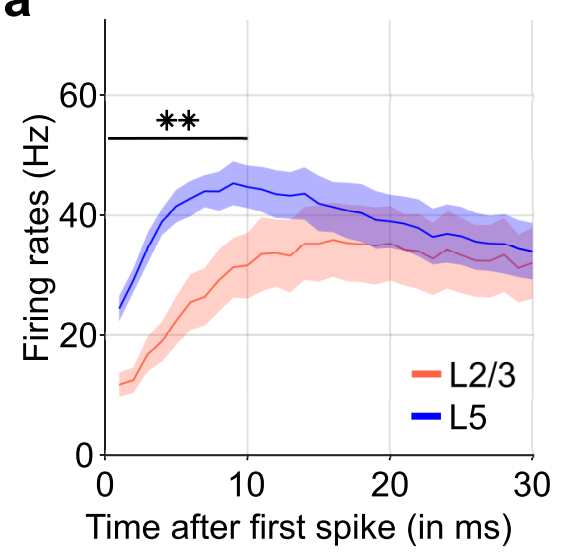

b

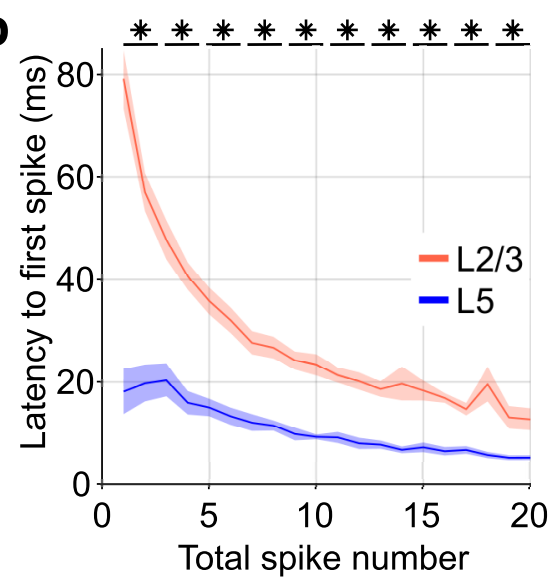

Extended Data Fig. 1 | Neuronal dynamics around OFF-ON transitions imply a leading role of layer 5 in population activity. a) Average neuronal firing activity at the transitions from population OFF to ON periods during baseline NREM sleep (layer $\times$ time interaction: $F(29,174)=9.412, p<0.001$, two-factor repeated measures ANOVA). Note that firing rates are higher in layer 5 during the first $10 \mathrm{~ms}$. b) Latency to the first spike for matched spike numbers during the first $200 \mathrm{~ms}$ of an ON period (main effect of layer: $F(1,6) 86.301, p<0.001$, two-factor repeated-measures ANOVA). Note that the latency to the first spike is shorter in layer 5 irrespective of the total number of spikes in a given ON period. $n=7$ wild type (C57L/6). Black asterisks indicate post-hoc contrasts with significant differences ( ${ }^{\star} p<0.05,{ }^{\star \star} p<0.01,{ }^{\star \star \star} p<0.001$ ). Data in panels $a, b$ are presented as mean \pm SEM (shaded areas). See Supplementary Table 1 for detailed results. L2/3, L5: Neocortical layers 2/3, 5. NREM: non-rapid eye movement sleep. 

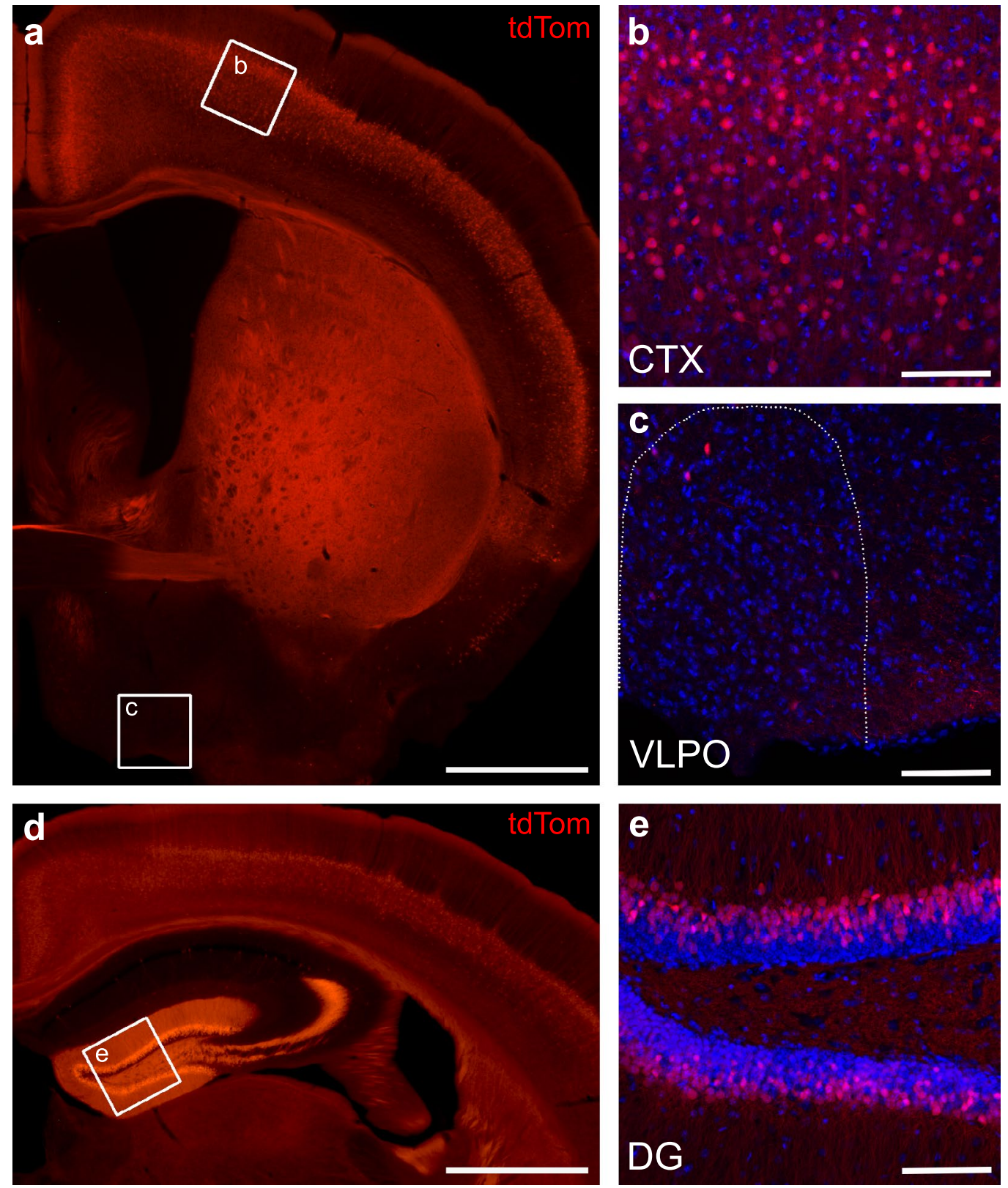

Extended Data Fig. 2 | Comparison of Cre-expression in the Rbp4-Cre mouse line between neocortical layer 5, dentate gyrus and hypothalamus. a) Coronal section of an Rbp4-Cre;Ai14;Snap25 $5^{1 /+}$ mouse brain indicating areas that were further examined for Cre-expression, using confocal imaging of DAPI stained slices. $\mathbf{b}, \mathbf{c})$ Laser scanning confocal microscope images from neocortex (CTX, b) and ventrolateral preoptic hypothalamus (VLPO, c) of DAPI stained (blue) sections, showing the distribution of tdTomato+ cells in the two regions. The VPLO region is outlined with a white, dotted line. Cell counts on corresponding coronal sections in three brains revealed that $20.53 \pm 0.98 \%(480 / 2342)$ of cortical L5 cells were tdTomato+, while only $1.15 \pm 0.40 \%$ (35/3006) of hypothalamic cells expressed the red fluorescent indicator. d) Coronal section of an Rbp4-Cre;Ai14;Snap25 $5^{f /+}$ mouse brain indicating the area of the dentate gyrus which was further examined for Cre-expression. e) Laser scanning confocal microscope image of dentate gyrus (DG) in a DAPI stained (blue) section. TdTomato+ cells were quantified in both the top and bottom blades of DG in three images, each from three different brains, and comprise $39.39 \pm 3.72 \%$ of cells in the granule layer. As evident from the boxed regions in $(a, d)$, the tdTomato+ cells in different brain regions vary in their fluorescence intensity, therefore the images in panels $(b, c, e)$ were acquired with settings optimised to show the tdTomato+ cells in each brain region. CTX: neocortex. DAPI: 4',6-diamidino-2-phenylindole. DG: dentate gyrus. VLPO: ventrolateral preoptic hypothalamus. Scale bars: $1 \mathrm{~mm}(\mathrm{a}, \mathrm{d}), 100 \mu \mathrm{m}$ (b,c,e). 

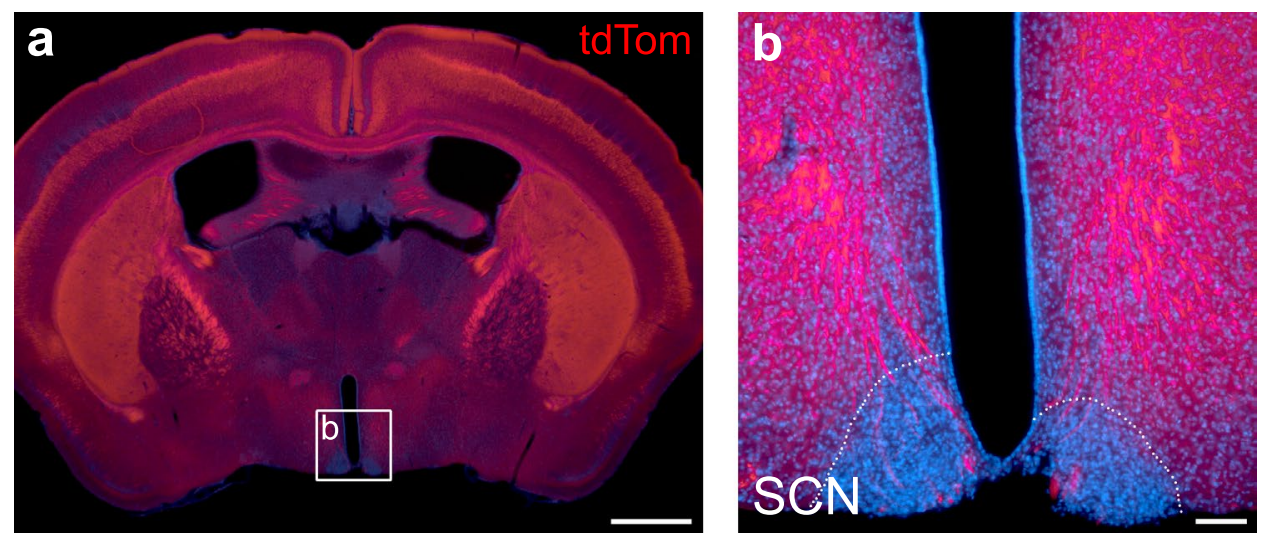

Extended Data Fig. 3 | The suprachiasmatic nucleus of the hypothalamus is void of Cre + cells and spared of fibre tracts in the Rbp4-Cre driver line. a) Epifluorescence image of an Rbp4-Cre;Ai14;Snap25 $5^{f /+}$ brain section at the level of the suprachiasmatic nucleus (SCN). The section was counterstained with DAPI (blue). Box indicates approximate region from which image in (b) was taken. b) High-magnification epifluorescence image of the SCN region. Rbp4-Cre;Ai14 axons are shown in red, cell nuclei stained with DAPI in blue. Note that there are no Cre+ cells located within the SCN (outlined with white dotted lines), and very few of the dense axon bundles pass through the SCN. DAPI: 4',6-diamidino-2-phenylindole. SCN: suprachiasmatic nucleus. Scale bars: $1 \mathrm{~mm}(\mathrm{a}), 100 \mu \mathrm{m}(\mathrm{b})$. 

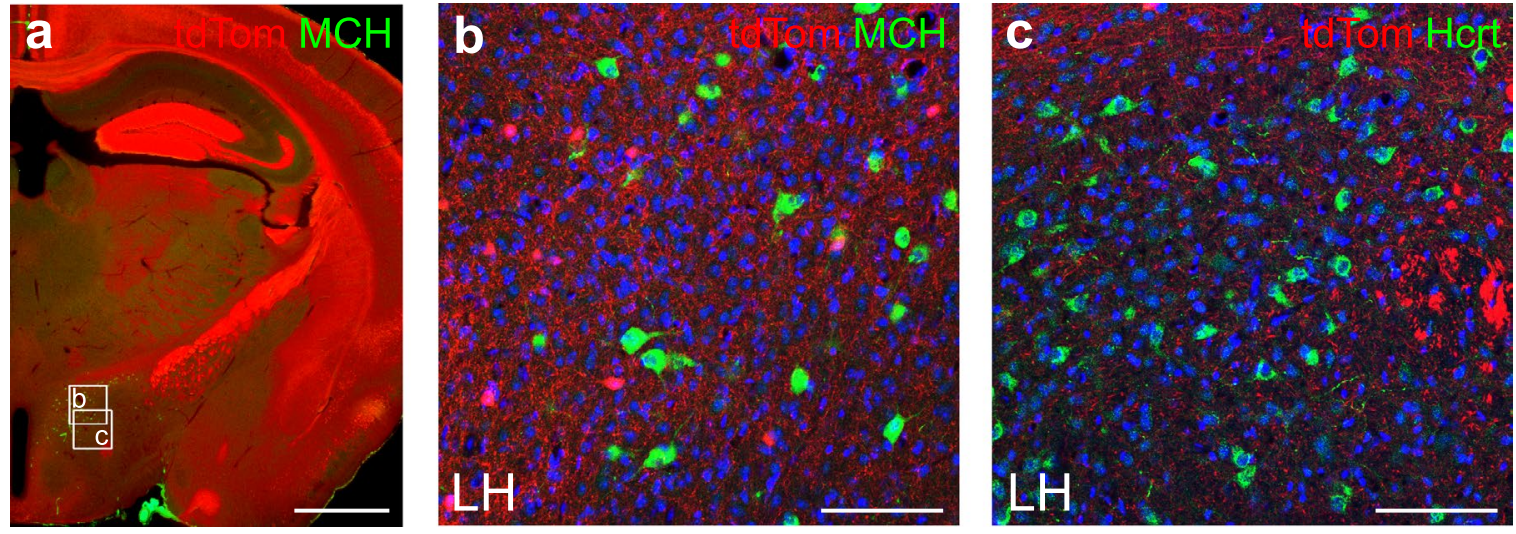

Extended Data Fig. 4 | No overlap between orexin or melanin concentrating hormone-expressing cells with Rbp4-Cre + cells but dense fibre tracts in lateral hypothalamus. a) Epifluorescence image of an Rbp4-Cre;Ai14;Snap $25^{f /+}$ brain hemisection at the level of the lateral hypothalamic area (LH), stained for melanin concentrating hormone $(\mathrm{MCH})$ in green. Boxes indicate approximate regions from which images in $(\mathbf{b}, \mathbf{c})$ were taken. $\mathbf{b}, \mathbf{c})$ Laser scanning confocal microscope images of two representative sections of LH of the same brain as shown in (a) stained for MCH (b) or orexin/hypocretin (Hcrt; $\mathrm{c}$ ). Rbp4-Cre;Ai14 cells and processes are shown in red, and nuclei are counterstained with DAPI (blue). Note that no $\mathrm{MCH}+\mathrm{cell} w a s \mathrm{tdTom}+(\mathrm{n}=3$ brains, $692 \mathrm{MCH}+$ cells $)$, and no Hcrt+ cell was tdTom $+(n=3$ brains, $469 \mathrm{Hcrt}+$ cells $)$. Note the dense fine fibres surrounding cell bodies in $\mathrm{LH}$, consistent with an axonal terminal field in that region. DAPI: 4',6-diamidino-2-phenylindole. Hcrt: orexin/hypocretin. MCH: melanin concentrating hormone. tdTom: tdTomato. Scale bars: $1 \mathrm{~mm}(\mathrm{a}), 100 \mu \mathrm{m}(\mathrm{b}, \mathrm{c})$. 


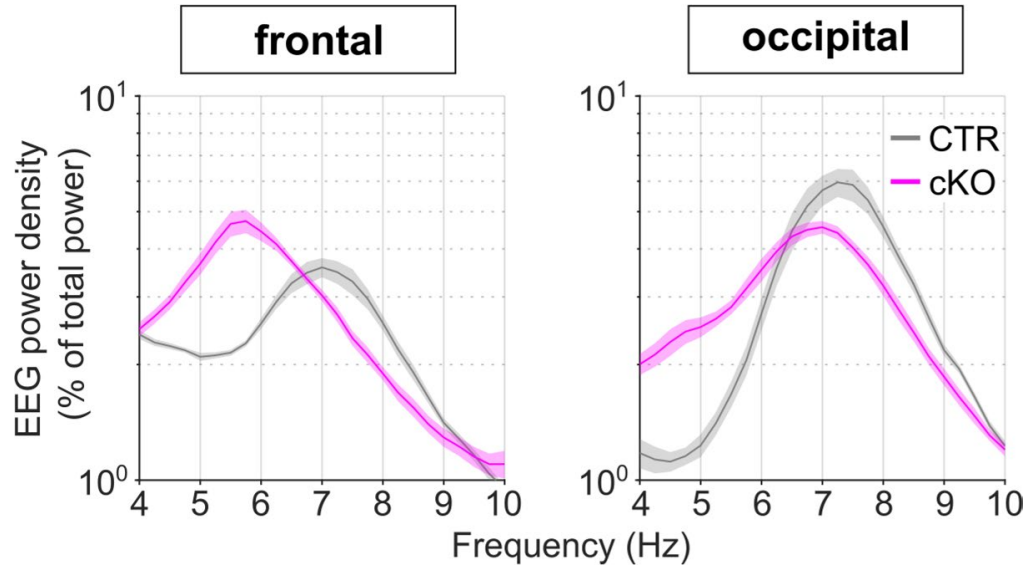

Extended Data Fig. 5 | The theta peak during REM sleep is shifted towards lower frequencies in cortical SNAP25-ablated mice. EEG spectral power in the frequency range $4-10 \mathrm{~Hz}$ normalised to the mean spectral power over the entire EEG spectrum $(0.5-30 \mathrm{~Hz})$ during REM sleep on the baseline day. Note that peak theta activity is shifted towards lower frequencies in cKOs compared to CTRs in both the frontal and occipital EEG derivations. $\mathrm{n}=5$ CTR and $n=8 \mathrm{cKO}$ for EEG spectral analysis. Data are presented as mean \pm SEM (shaded areas). See Supplementary Table 1 for detailed results. cKO: conditional knockout animals. CTR: control animals. EEG: Electroencephalogram. REM: Rapid eye movement sleep. 


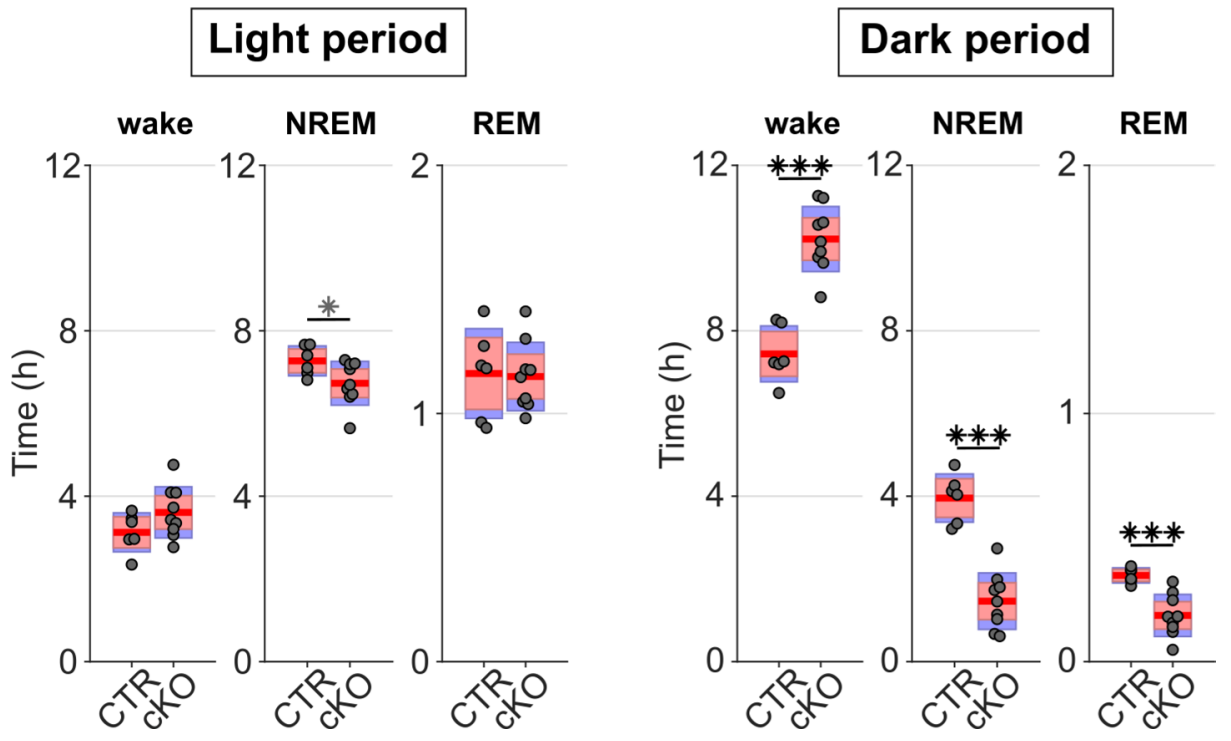

Extended Data Fig. 6 | Genotype differences in the amount of time spent in wake, NREM, and REM sleep during undisturbed baseline recordings are more pronounced in the dark period. During the light period, the distribution of vigilance states is similar between genotypes with only a trend towards increased wakefulness and reduced NREM and REM sleep, while strong differences occur during the dark period (genotype $\times$ phase $\times$ vigilance state interaction: $F(1,14)=36.083, p<0.001$, three-way ANOVA). $n=6$ CTR and $n=9$ cKO for vigilance state analysis. Black asterisks indicate post-hoc contrasts with significant differences $\left({ }^{*} p<0.05,{ }^{* *} p<0.01,{ }^{\star *} p<0.001\right)$, grey asterisks indicate post-hoc comparisons with $P<0.05$, which do not reach significance after Bonferroni correction for multiple comparisons. Data is presented as group mean (red line), $95 \%$ confidence interval (pink box), and one standard deviation (blue box) with individual data points overlaid. See Supplementary Table 1 for detailed results. cKO: conditional knockout animals. CTR: control animals. NREM: Non-rapid eye movement sleep. REM: Rapid eye movement sleep. 

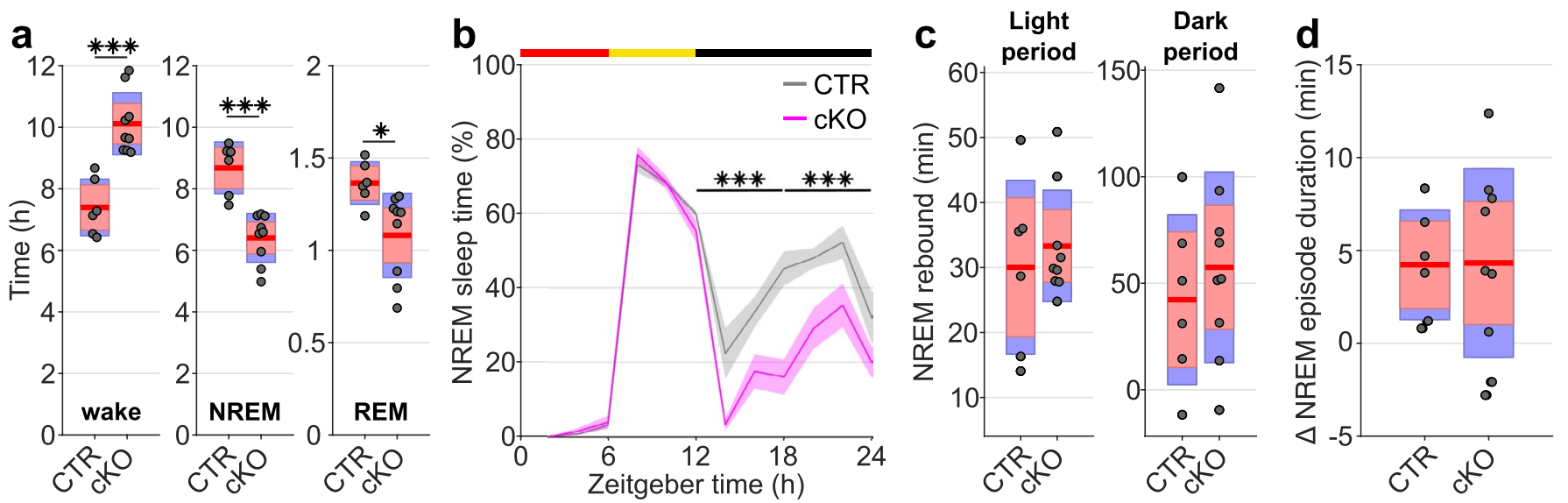

Extended Data Fig. 7 | Absolute time in NREM sleep following sleep deprivation is reduced in cortical SNAP25-ablated animals but relative NREM rebound does not differ between genotypes. a) Time spent in vigilance states (wake, NREM, and REM) during the $18 \mathrm{~h}$ recovery time following sleep deprivation (genotype $\times$ vigilance state interaction: $F(1,14)=27.754, p<0.001$, mixed ANOVA). Note that cortical SNAP25-ablated animals (cKO) overall spent more time awake and less time in NREM and REM sleep compared to controls (CTR). b) Time course of NREM sleep on a sleep deprivation day compared between genotypes (genotype $\times$ time interaction: $F(4,54)=4.222, p=0.004$, mixed ANOVA). cKOs sleep less during the entire $12 \mathrm{~h}$ dark period following sleep deprivation. c) Rebound of NREM sleep time following sleep deprivation relative to individual baseline values. No differences were observed between genotypes. d) Change in duration of NREM episodes during the first hour after sleep deprivation (ZT6-7 of SD day) relative to the same time window on BL day. $n=6$ CTR and $n=9 \mathrm{CKO}$ for vigilance state analysis. Black asterisks indicate post-hoc contrasts with significant differences $\left({ }^{\star} p<0.05,{ }^{\star \star} p<0.01,{ }^{\star \star *} p<0.001\right)$. Data in panels a, $c, d$ is presented as group mean (red line), 95\% confidence interval (pink box), and one standard deviation (blue box) with individual data points overlaid. Data in panel b are presented as mean values \pm SEM(shaded areas). See Supplementary Table 1 for detailed results. BL: baseline. cKO: conditional knockout animals. CTR: control animals. NREM: Non-rapid eye movement sleep. REM: Rapid eye movement sleep. SD: sleep deprivation. ZT: zeitgeber time. 
a

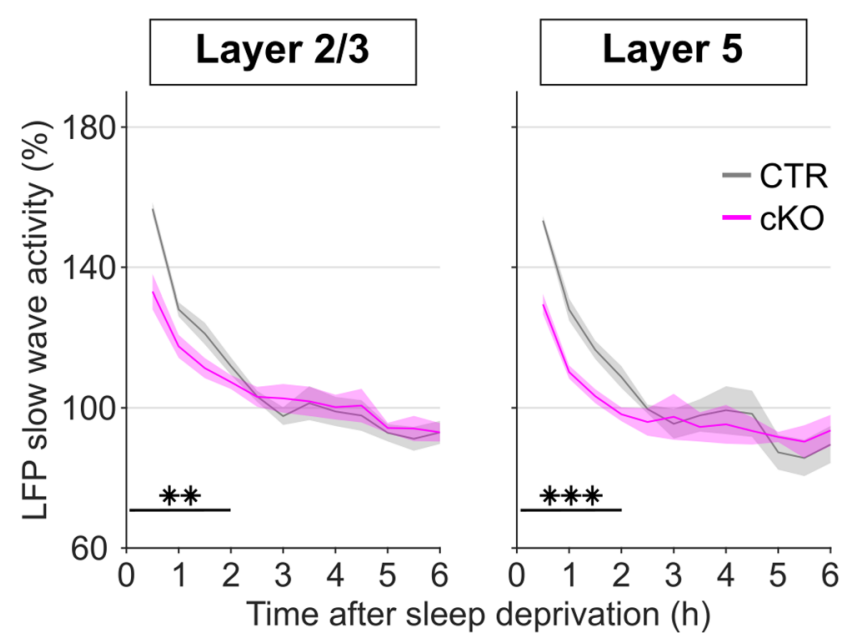

b

EEG recordings

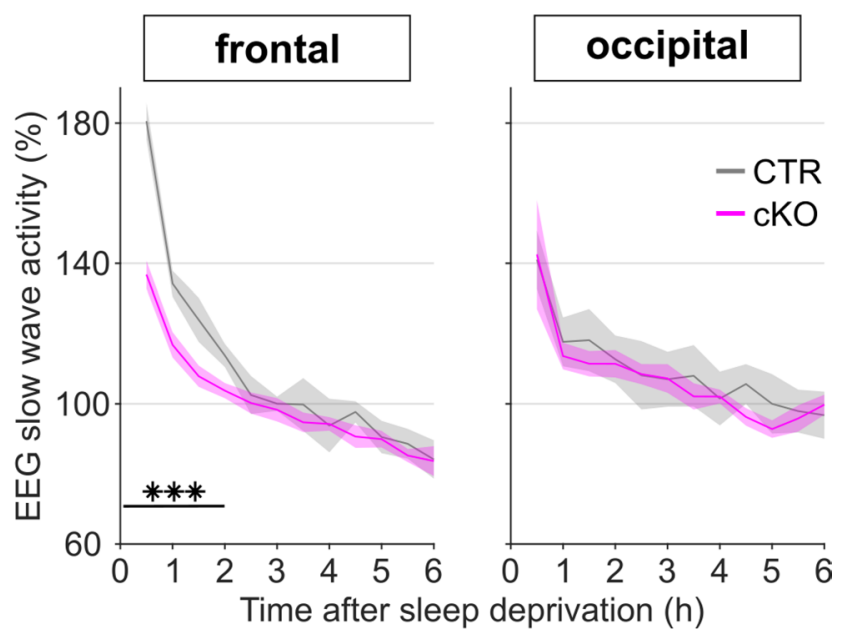

Extended Data Fig. 8 | The rebound of slow wave activity after sleep deprivation is specific to cortical areas but not layers. Time course of NREM slow wave activity (SWA) after sleep deprivation (a) in the LFPs from layers $2 / 3$ and 5 in primary motor cortex and (b) in the frontal and occipital EEG derivation. Note that cortical SNAP25-ablated animals (cKO) had lower initial SWA levels in the frontal EEG and LFP recordings across all layers, compared to controls (CTR). $n=5$ CTR and $n=5$ cKO for laminar analysis, $n=5$ CTR and $n=8$ cKO for EEG spectral analysis. Black asterisks indicate post-hoc contrasts with significant differences $\left({ }^{\star} p<0.05,{ }^{\star \star} p<0.01,{ }^{\star \star \star} p<0.001\right.$ ). Data in panels a and $b$ are presented as mean values \pm SEM (shaded areas). See Supplementary Table 1 for detailed results. cKO: conditional knockout animals. CTR: control animals. 


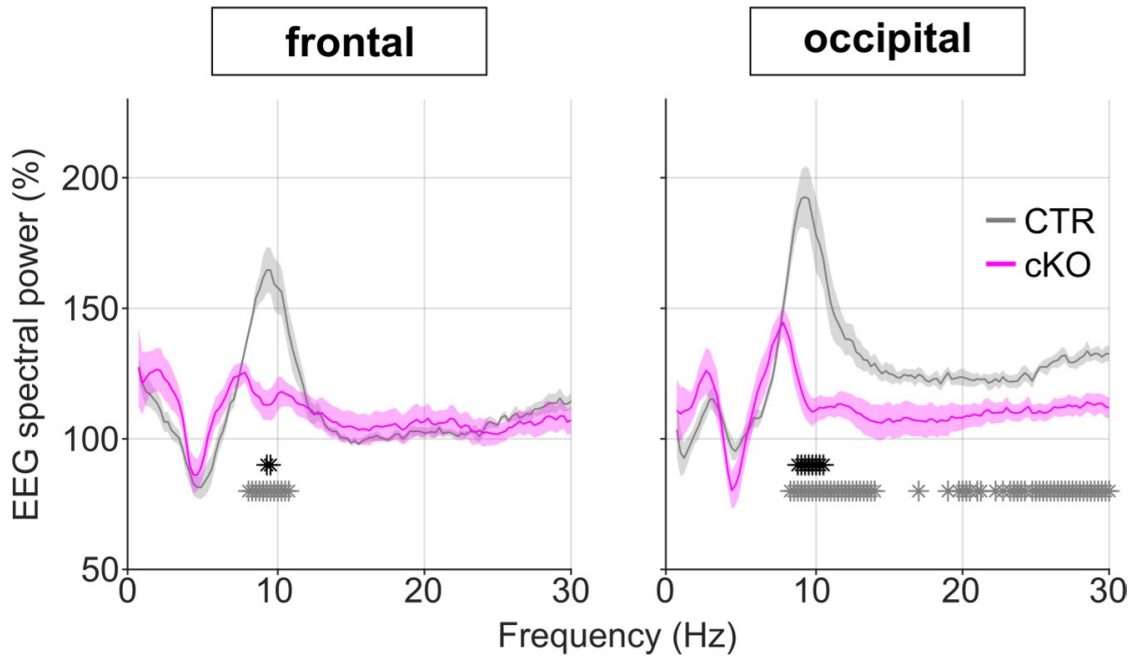

Extended Data Fig. 9 | Relative EEG power spectra during sleep deprivation show an attenuated increase in theta activity cortical SNAP25-ablated animals. Wake EEG spectral power during the 6-hour sleep deprivation shown as a frequency bin-wise percentage of $24 \mathrm{~h}$ baseline values. Note that the expected increase in theta-power during sleep deprivation, which is visible in CTR mice, is severely diminished in $c K O s . n=5$ CTR and $n=8 \mathrm{cKO}$ for EEG spectral analysis. Individual asterisks indicate spectral bins with significant differences in post-hoc comparison before (grey) and after (black) Bonferroni adjustment of $\alpha$. Data are presented as mean values \pm SEM (shaded areas). See Supplementary Table 1 for detailed results. cKO: conditional knockout animals. CTR: control animals. EEG: electroencephalogram. 

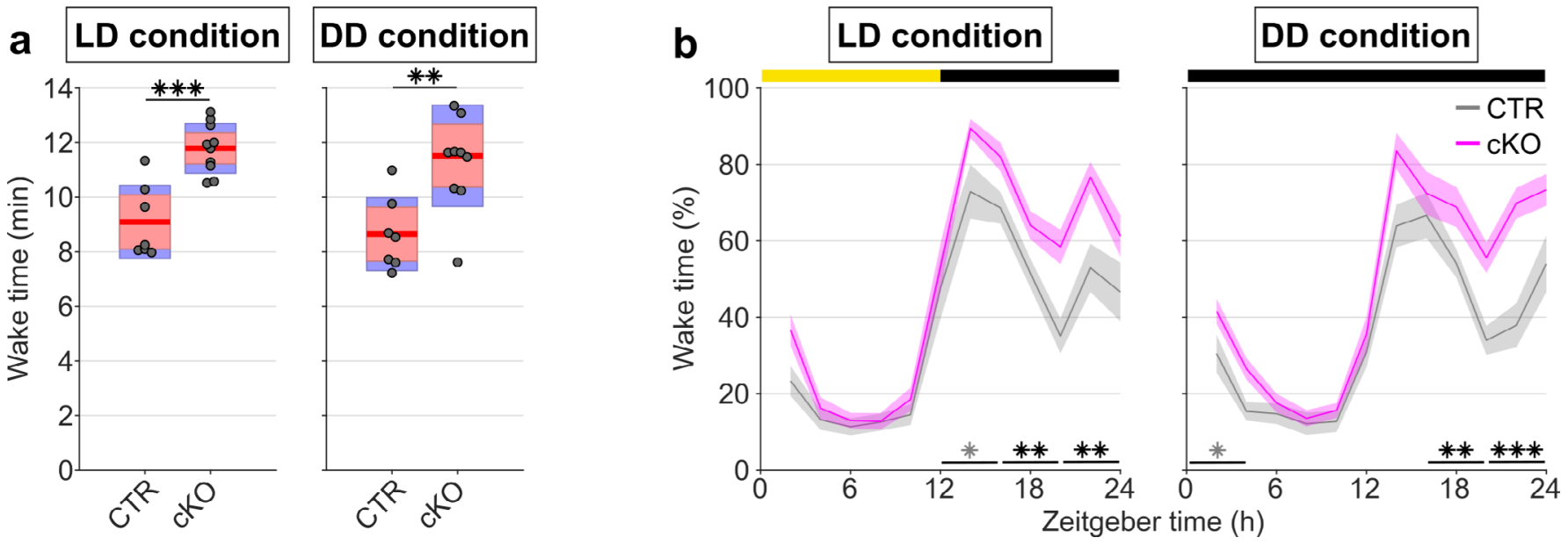

Extended Data Fig. 10 | Passive infrared recordings (PIR) show robustness of sleep phenotype to altered light-conditions. a) Wake time estimates averaged over the last 3 days of PIR recordings under 12:12 light-dark (LD) conditions and over the first 3 days of constant darkness (DD) (main effect of genotype: $F(1,15)=18.604, p=0.001$, mixed ANOVA). Note that genotype differences in the daily amount of wakefulness persist in the absence of light. b) Time course of wakefulness in LD and DD conditions (genotype $x$ time interaction: $F(3.801,57.016)=3.319, p=0.018$, mixed ANOVA). $n=7$ CTR and $n=10$ for PIR recordings. cKO: conditional knockout animals. Black asterisks indicate post-hoc contrasts with significant differences $\left({ }^{*} p<0.05,{ }^{* *} p<0.01\right.$, ${ }^{\star \star *} p<0.001$ ), grey asterisks indicate post-hoc comparisons with $P<0.05$, which do not reach significance after Bonferroni correction for multiple comparisons. Data in panel a is presented as group mean (red line), $95 \%$ confidence interval (pink box), and one standard deviation (blue box) with individual data points overlaid. Data in panel $b$ are presented as mean values \pm SEM (shaded areas). See Supplementary Table 1 for detailed results. CTR: control animals. LD: light:dark. DD: constant darkness. PIR: passive infrared recordings. 


\section{Reporting Summary}

Nature Research wishes to improve the reproducibility of the work that we publish. This form provides structure for consistency and transparency in reporting. For further information on Nature Research policies, seeAuthors \& Referees and theEditorial Policy Checklist.

\section{Statistics}

For all statistical analyses, confirm that the following items are present in the figure legend, table legend, main text, or Methods section.

n/a Confirmed

$\square \quad \boldsymbol{x}$ The exact sample size ( $n$ ) for each experimental group/condition, given as a discrete number and unit of measurement

$\square x$ A statement on whether measurements were taken from distinct samples or whether the same sample was measured repeatedly

$\boldsymbol{x}$ The statistical test(s) used AND whether they are one- or two-sided

x Only common tests should be described solely by name; describe more complex techniques in the Methods section.

$\mathbf{x} \square$ A description of all covariates tested

x A description of any assumptions or corrections, such as tests of normality and adjustment for multiple comparisons

$\boldsymbol{x}$ A full description of the statistical parameters including central tendency (e.g. means) or other basic estimates (e.g. regression coefficient)

$\boldsymbol{x}$ AND variation (e.g. standard deviation) or associated estimates of uncertainty (e.g. confidence intervals)

x For null hypothesis testing, the test statistic (e.g. $F, t, r$ ) with confidence intervals, effect sizes, degrees of freedom and $P$ value noted

$\boldsymbol{x}$ Give $P$ values as exact values whenever suitable.

$\mathbf{x} \square$ For Bayesian analysis, information on the choice of priors and Markov chain Monte Carlo settings

$\boldsymbol{x} \square$ For hierarchical and complex designs, identification of the appropriate level for tests and full reporting of outcomes

$\square \boldsymbol{x}$ Estimates of effect sizes (e.g. Cohen's $d$, Pearson's $r$ ), indicating how they were calculated

our web collection on statistics for biologists contains articles on many of the points above.

\section{Software and code}

Policy information about availability of computer code

Data collection

The software package 'Synapse Suit' (Tucker-Davis Technologies Inc., Alachua, FL, USA) was used for data collection.

Data analysis

Custom-made code in Matlab (The MathWorks Inc, Natick, Massachusetts, USA, version v2017a) was used for resampling of rawdata and for further electrophysiological data analysis. Commented Matlab code for key analyses is deposited on Figshare (DOI: 10.6084/ m9.figshare.14737578). The software Sleep Sign for Animals (SleepSign Kissei Comtec Co., Ltd., Nagano, Japan, version 3.3.6.1602) was used for sleep scoring and to compute the fast Fourier transform routine. IBM SPSS Statistics for Windows (IBM Corp., Armonk, N.Y., USA, version 25.0) and SAS JMP (SAS Institute Inc. Cary, NC, USA, version 7.0) were used for statistical analysis. ImageJ (Wayne Rasband, National Institutes of Health, USA, version 1.52a) was used to merge fluorescent images and add scale bars. The ImageJ plug-in "ActogramJ" (Benjamin Schmid and Taishi Yoshii, University of Wurzburg, Germany) was used for periodogram analysis. Figures were prepared in Inkscape (Inkscape project, version 1.0.2).

For manuscripts utilizing custom algorithms or software that are central to the research but not yet described in published literature, software must be made available to editors/reviewers. We strongly encourage code deposition in a community repository (e.g. GitHub). See the Nature Research guidelines for submitting code \& software for further information. 
Policy information about availability of data

All manuscripts must include a data availability statement. This statement should provide the following information, where applicable:

- Accession codes, unique identifiers, or web links for publicly available datasets

- A list of figures that have associated raw data

- A description of any restrictions on data availability

Statistical source data for all figures is available for download via the Nature Neuroscience weblink to this paper. Imaging source data is deposited on Figshare (DOI: $10.6084 / \mathrm{m} 9$. figshare.14737584). A sample dataset with spectral data and sleep scoring results used to generate key analyses presented in this manuscript is available for download on Figshare (DOI: 10.6084/m9.figshare.14737569). Raw data from electrophysiological and passive infrared recordings are available from the corresponding authors upon reasonable request.

\section{Field-specific reporting}

Please select the one below that is the best fit for your research. If you are not sure, read the appropriate sections before making your selection.
$\mathbf{x}$ Life sciences
Behavioural \& social sciences
Ecological, evolutionary \& environmental sciences

For a reference copy of the document with all sections, see nature.com/documents/nr-reporting-summary-flat.pdf

\section{Life sciences study design}

All studies must disclose on these points even when the disclosure is negative.

Sample size Sample sizes were initially based on previous studies (Huber et al., Brain Research, 2000, and Vyazovskiy et al., Nature, 2011). Power calculations were performed after acquisition of pilot data for the Wellcome Trust grant proposal 203971/Z/16/A indicated an effect size of $d$ $=2.75$ for the main outcome parameter NREM sleep time over 24 hours. Applying an intended power of 0.9 and an alpha-error probability of 0.01 , the power calculations confirmed the initial sample size estimate of $n=8$ animals per genotype considering an attrition rate of $25 \%$.

Data exclusions No animals or data were excluded. The exact number of animals contributing to specific experiments/analyses is always mentioned in the text and/or figure legends.

$\begin{array}{lll}\text { Replication } & \text { The key finding of this study was confirmed in two independent cohorts of animals, using two different recording methods }\end{array}$ (electroencephalography and passive infrared recordings).

Randomization Animals were retrieved from the breeder colony upon availability. Based on the breeding scheme we expected that male Rbp4Cre;Ai14;Snap25f//fl mice only represent on average $1 / 8$ of the animals in each litter. As soon as animals of the desired genotype were available, they were included in the study and the recording capacities were filled with randomly selected Cre-negative controls from the colony, preferably littermates.

Blinding The experimenters were blinded to the genotype of the transgenic animals in all experiments performed with passive infrared recordings. The experimenters were not blinded during the electrophysiological experiments. Sleep scoring could not be performed fully blinded as originally intended because the characteristic features of increased amount of wakefulness and long wake episodes allowed a clear distinction between the two genotypes during the scoring process.

\section{Reporting for specific materials, systems and methods}

We require information from authors about some types of materials, experimental systems and methods used in many studies. Here, indicate whether each material, system or method listed is relevant to your study. If you are not sure if a list item applies to your research, read the appropriate section before selecting a response.

\begin{tabular}{l|l} 
Materials \& experimental sys \\
\hline n/a & Involved in the study \\
\hline & $\boldsymbol{x}$ Antibodies \\
\hline $\boldsymbol{x}$ & $\square$ Eukaryotic cell lines \\
\hline $\boldsymbol{x}$ & $\square$ Palaeontology \\
\hline$\square$ & $\boldsymbol{x}$ Animals and other organisms \\
\hline $\boldsymbol{x}$ & $\square$ Human research participants \\
$\boldsymbol{x}$ & $\square$ Clinical data
\end{tabular}

\begin{tabular}{|c|c|}
\hline \multicolumn{2}{|c|}{ Methods } \\
\hline $\mathrm{n} / \mathrm{a}$ & Involved in the study \\
\hline$x$ & $\square$ ChIP-seq \\
\hline$x$ & $\square$ Flow cytometry \\
\hline$x$ & $\square$ MRI-based neuroimaging \\
\hline
\end{tabular}

\section{Antibodies}


All antibodies have been used and validated in previous studies.

Validation of the anti-MCH antibody is reported in: Hanriot, L. et al. Characterization of the melanin-concentrating hormone neurons activated during paradoxical sleep hypersomnia in rats. J. Comp. Neurol. 505, 147-157 (2007).

Validation of the anti-Hcrt antibody is reported in: van den Pol, A. N., et al. Presynaptic and Postsynaptic Actions and Modulation of Neuroendocrine Neurons by a New Hypothalamic Peptide, Hypocretin/Orexin. J. Neurosci. 18, 7962 LP - 7971 (1998).

\section{Animals and other organisms}

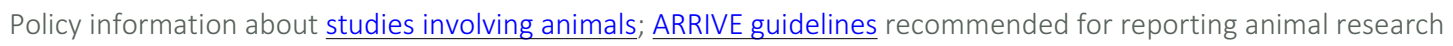

Laboratory animals

Electrophysiology experiments:

7 wild type C57BL/ 6 mice (age at baseline recording $125 \pm 8$ days)

12 Rbp4-Cre;Ai14;Snap25fl/fl mice (age at baseline recording 90 \pm 5 days) and 8 Cre-negative littermates (age at baseline recording $85 \pm 4$ days)

Passive infrared recordings:

10 Rbp4-Cre;Ai14;Snap25fl/fl mice (age at baseline recording $84 \pm 1$ days) and 7 Cre-negative littermates (age at baseline recording $83 \pm 1$ days).

Anatomical work:

6 Rbp4-Cre;Ai14;Snap25fl/+ mice (age at perfusion data $57 \pm 6$ days)

All experiments were conducted in young adult male mice. A 12:12 h light:dark cycle (lights on at 9 am, light levels 120-180 lux) was implemented, temperature maintained at around $22 \pm 2^{\circ} \mathrm{C}$, and humidity kept around $50 \pm 20 \%$.

Wild animals

Field-collected samples

Ethics oversight
No wild animals were used in this study.

The study did not involve samples collected from the field.

All experiments were performed in accordance with the United Kingdom Animal Scientific Procedures Act 1986 under persona and project licences granted by the United Kingdom Home Office. Ethical approval was provided by the Ethical Review Panel at the University of Oxford.

Note that full information on the approval of the study protocol must also be provided in the manuscript. 